\title{
Addressing some misconceptions regarding seismic hazard assessment in mines
}

\author{
J. Wesseloo \\ Australian Centre for Geomechanics, The University of Western Australia
}

\begin{abstract}
Seismic hazard assessment, in some form or another, has formed part of seismic risk management in seismically active hard rock mines for decades. Whilst recent advances have been made in seismic hazard assessment, some misconceptions with respect to seismic hazard assessment appear to be present in the industry. Where these misconceptions exist, they adversely affect the quality of seismic hazard assessment and risk management decision-making. This paper address some of these misconceptions the author has encountered in the mining industry.
\end{abstract}

\section{INTRODUCTION}

At any seismically active mine, considerable effort is invested into the effective management of seismic risk (see Potvin et al., 2019), of which seismic hazard assessment is, of course, a fundamental component. Over many years I have come across several misconceptions regarding the assessment of the seismic hazard which adversely affects the standard of seismic risk management in our industry. Some of these misconceptions are widespread and deeply rooted, whilst others crop up from time to time and seem to migrate through the industry. The aim of this paper is to address some of those misconceptions.

The frequency-magnitude (FM) distribution is a foundational building block in understanding seismic hazard. It appears, however, that many of the misunderstandings regarding seismic hazard flow from inadequate understanding of the meaning and implications of the FM distribution. For this reason, a large proportion of this paper will be devoted to the FM distribution and its implications. The fact that ' $M_{\max }$ ' is used for several different concepts, further creates confusion and misunderstanding. Another topic that does not seem to be well understood is that of normalisation of hazard, with respect to space and time, and the related issue of separating sources of seismicity with different behaviour.

\section{WHAT IS THE GUTENBERG-RICHTER RELATIONSHIP REALLY?}

It appears that many misconceptions in the mining industry stem from an inadequate understanding of the FM distribution and its implications. Many rock engineers use the FM chart without realising that it is simply a reverse cumulative distribution ${ }^{1}$ of magnitude, with the vertical axis plotted on a log scale. The straight line fit, or any other curve fitted to the data, is therefore simply a statistical best-fit model and is conceptually the same as, for example, a normal distribution fitted to UCS data (See Figure 1).

\footnotetext{
${ }^{1}$ Also referred to as Complimentary Cumulative Distribution of Inverse Cumulative Distribution. The term ‘nverse Cumulative Distribution', however, is also used to refer to the quantile function.
} 

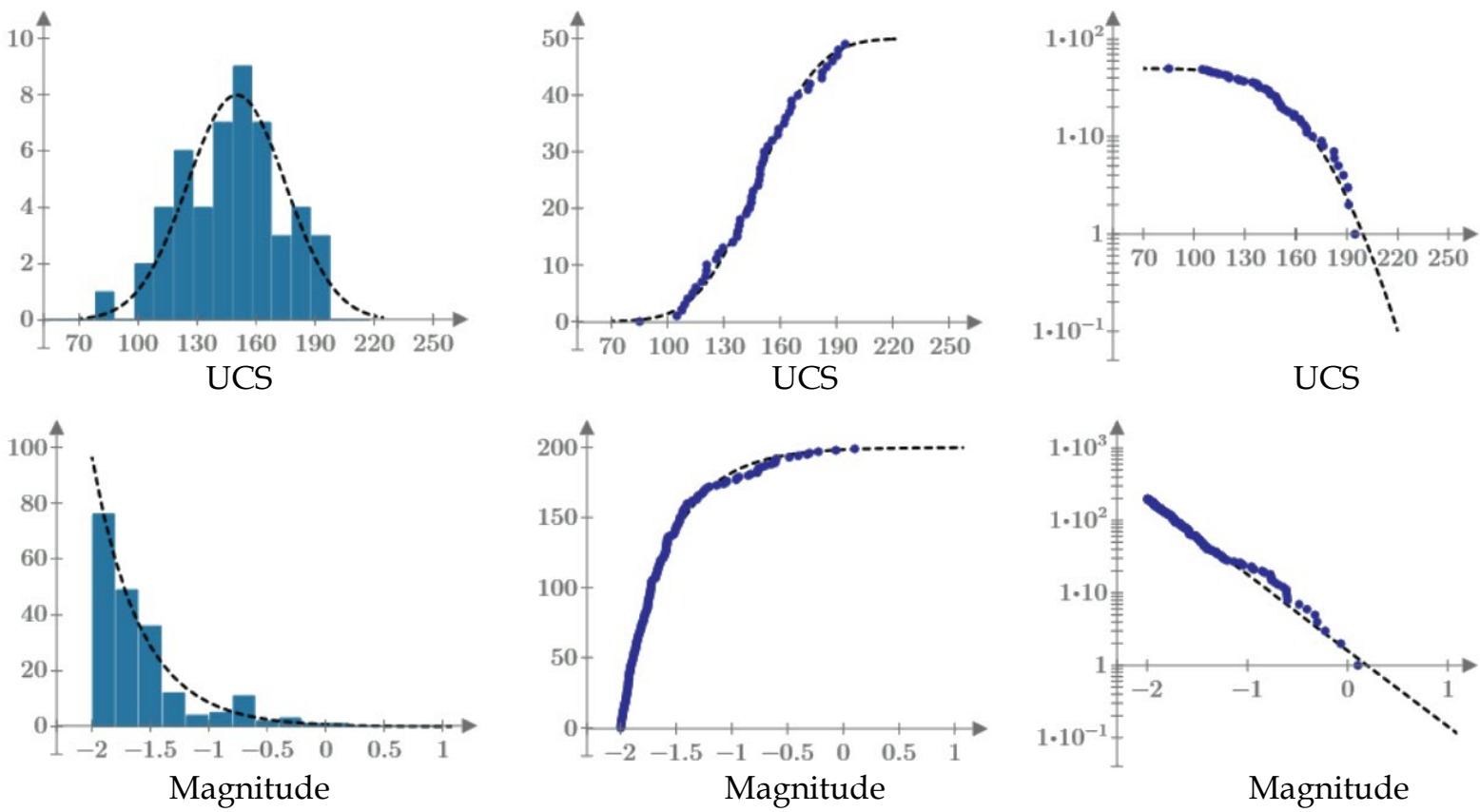

a) Histogram

b) Cumulative distribution

c) Reverse cumulative distribution

Figure 1. Graphical representation and the statistical best fit model of UCS data and seismic magnitude data

The model most often used for seismic magnitude distribution is the Gutenberg-Richter (GR) relationship (Gutenberg and Richter, 1944):

$$
\log (N)=a-b \cdot M
$$

Which we can write as:

$$
N=10^{a-b \cdot M}
$$

where:

$N=$ the number of events with magnitude $>=M$

$M=$ event magnitude

$a=$ coefficient quantifying the number of events

$b=$ coefficient quantifying the log relative occurrence of smaller to larger events

In the case of seismic magnitude data, however, limitations in the system sensitivity results in small events not being recorded. For statistical and probabilistic analysis, one can therefore only use the data above the magnitude of completeness, $m_{m i n}$, with the statistical density and cumulative function being limited to the magnitude ranges greater than $m_{\min }$. We can write the GR relationship in Equation 2, with respect to the reverse cumulative distribution function, as follows: 


$$
N=N_{\min } \cdot F^{\prime}(M)
$$

with

and

$$
F^{\prime}(M)=\left\{\begin{aligned}
1, & M<m_{\text {min }} \\
10^{-b\left(M-m_{\text {min }}\right)}, & M \geq m_{\text {min }}
\end{aligned}\right.
$$

$$
N_{\text {mmin }}=10^{a-b \cdot m_{\text {min }}}
$$

Where

$N_{\text {mmin }}=\quad$ the number of events with magnitude greater than $m_{\min }$

$F^{\prime}(M)=$ the reverse cumulative distribution function

It is interesting to note that $\mathrm{F}^{\prime}(\mathrm{M})$ in Equation 2 is simply a different formulation of the commonly used negative exponential distribution, translated to start at $m_{\min }$ instead of 0 .

The open-ended Gutenberg-Richter (GR) relationship (Equations 1 and 2) predicts a non-finite probability of physically impossible magnitude sizes, (i.e., the probability of a Richter magnitude $>10$ is not zero!). For this reason, equations that truncate at large magnitudes are preferred of which several different relationships have been proposed (Utsu, 1999). In mining, the truncated Gutenberg-Richter (TGR) relationship (Page, 1968) is often used. Equation 3 rewrites Page's formulation to follow the general formulation that is commonly used by rock engineers, as presented in Equation 3.

$$
N=N_{\min } \cdot F^{\prime}(M)
$$

With the reverse cumulative distribution written as:

$$
F^{\prime}(M)=\left\{\begin{aligned}
1, & \mathrm{M}<m_{\text {min }} \\
1-\frac{1-10^{-b\left(M-m_{\min }\right)}}{1-10^{-b\left(M_{U L}-m_{\min }\right)},}, & m_{\min } \leq \mathrm{M}<M_{U L} \\
0, & \mathrm{M} \geq M_{U L}
\end{aligned}\right.
$$

Where

$N_{\text {mmin }}=\quad$ the number of events with magnitude greater than $m_{\text {min }}$ $M_{U L} \quad=\quad$ the upper truncation magnitude of the distribution

Similar to Equation 2, Equation 4 is a different formulation of a truncated and translated negative exponential distribution.

The cumulative distribution function $F(M)$ and the probability density functions for the TGR is as follows:

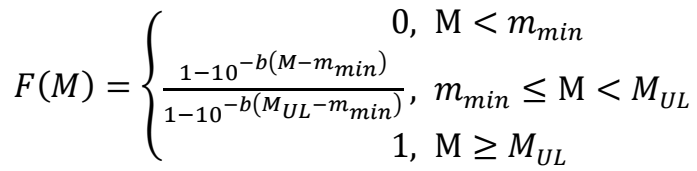

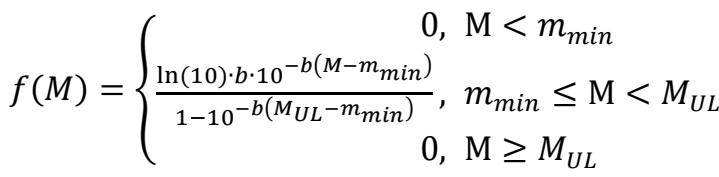




\section{THE M MAX CONFUSION}

In the mining industry, the term ' $M_{\max }$ ' is used for several different concepts, with the loose meaning of 'expected largest event' associated with it. These concepts are (See Figure 2):

a) the largest recorded event in a dataset

b) the value of the fitted GR relationship at $N=1$

c) the distribution of the largest expected magnitude

d) The largest physical possible event

e) The upper asymptote of the FM distribution.

To avoid confusion, we refer to each of these by a separate name: a) $X_{\max }$, b) $a / b$, and c) $f_{\max }$, d) $M_{\max }$, and e) $M_{u L}$. Each of these are, in some way or another, being used as hazard indicators and are discussed in the following paragraphs. Generally, no distinction is made between $M_{\max }$ and $M_{u L}$, and the two concepts are mostly used interchangeably. Conceptually $M_{\max }$ has a physical meaning, while $M_{u L}$ carries only the abstract meaning of being an upper limit of a probability distribution. I find it useful to keep these two concepts separated and will discuss this in more detail further on in the paper.

Due to the stochastic nature of seismicity, the largest event magnitude within a given number of events is a distribution and cannot be captured with a single number. This distribution is represented as a colour spectrum at $N=1$ in Figure 2 . We refer to this distribution as $f_{\max }, F_{\max }$ and $F_{\text {max }}^{\prime}$ for the probability density, cumulative probability and reverse cumulative probability functions, respectively. It is important to note that any statement regarding the expectation of specified event magnitudes is dependent on the number of events. For any such statement to be meaningful, it is necessary to specify the number of events applicable to this statement. In this paper, the short notation, N@M, is used; for example, $\mathrm{N} @-1=100$ or $\mathrm{N} @ 0=1$, and the $a$-value in Equations 1 to 4 is equal to $\log (\mathrm{N} @ 0)$.

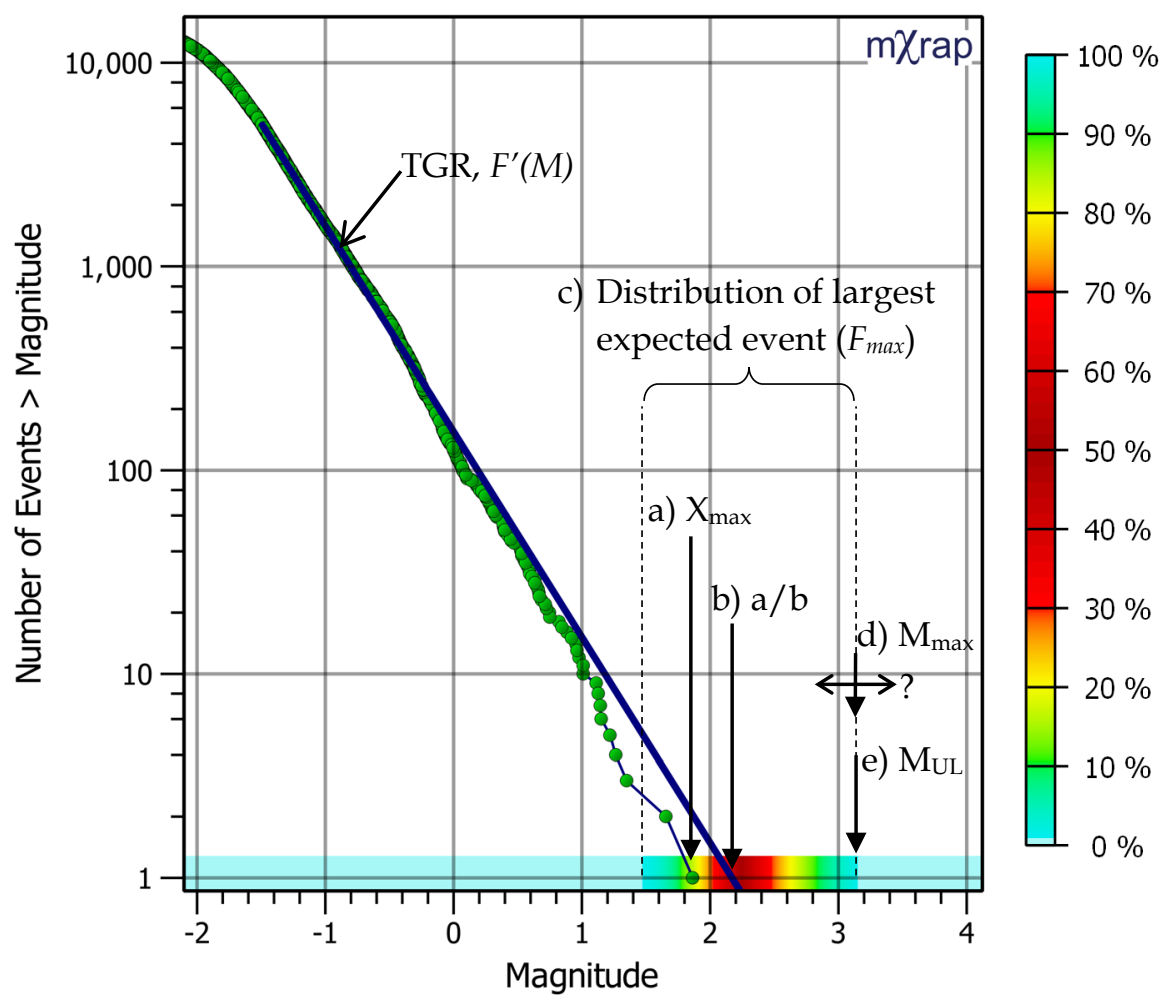

Figure 2. FM distribution of seismic magnitude 


\section{DISTRIBUTION OF THE EXPECTED LARGEST EVENT}

The two $a / b$ and $M_{U L}$, shown in Figure 2 are important parameters in the assessment and communication of seismic hazard. It appears though, that undue emphasis is sometimes placed on these parameters which does not fully capture the seismic hazard. Consider the distribution of the maximum expected event within N@-1.5 = 4000, shown in Figure 2, the value of $M_{u L}$ defines only the upper limit of the $F(M)$ and $F_{\max }(M)$ distributions and the $a / b$ value describes only one point on those distributions. The whole body of the distribution, however, describes the hazard.

The distribution of the expected largest event can be obtained directly from the FM distribution. The cumulative distribution function of the largest magnitude within $n$ events is given by (Gibowicz and Kijko, 1994):

where:

$$
F_{\text {max }}(M, n)=(F(M))^{n}
$$

$F_{\max }(M, n)=$ cumulative distribution function of the magnitude of the largest event within $\mathrm{n}$ events $n=$ the number of events with magnitude $\geq m_{\min }$

The probability density function of the largest event within $n$ events can be obtained as the derivative of $F_{\max }$ and is as follows:

$$
f_{\text {max }}(M, n)=\left\{\begin{array}{cl}
0, & M<m_{\text {min }} \\
n \cdot f(M) \cdot F(M)^{n-1}, & m_{\min } \leq \mathrm{M}<M_{U L} \\
0, & \mathrm{M} \geq M_{U L}
\end{array}\right.
$$

where:

$f_{\max }(M, n)=$ probability density functions of the magnitude of the largest event within $\mathrm{n}$ events

$F(M), f(M)=$ the cumulative distribution and probability density functions of the FM distribution (e.g. Equation 5 and 6$)$

$n$

$=\quad$ the number of events with magnitude $\geq m_{\min }$

It is well-known that the intensity of dynamic waves attenuates sharply with distance. Using the Canadian Rockburst Handbook formulation (Mining Research Directorate, 1996), one can estimate that the body wave peak particle velocity (ppv) resulting from a $\mathrm{M}_{\mathrm{L}} 3$ at a distance of $500 \mathrm{~m}$ is similar to that from a $\mathrm{M}_{\mathrm{L}} 0.5$ at a distance of $20 \mathrm{~m}$. Assuming a $b$-value of 1 , one would expect about 315 events of $\mathrm{M}_{\mathrm{L}}>0.5$ for every single event of $\mathrm{M}_{\mathrm{L}}>3$. When considering the fact that the workforce is exposed to the occurrence of many more small events at close proximity, it is clear that the body of the distribution and not only the upper limit or the mode of the distribution is important. This is evidenced in the fact that it is becoming more common to install face support in development headings to protect the workforce against the effect of events much smaller than the $M_{u L}$.

The consequence of smaller events is, of course, expected to be much smaller than that of an $M_{\max }$ event. One useful way to quantify the hazard, therefore, is to express it as the probability of exceeding a large event, for example $M_{L} 1, M_{L} 2$ and $M_{L} 2.5$ is commonly used, which for the illustration in Figure 2 is about $100 \%, 70 \%$ and $30 \%$ respectively.

\section{$\mathbf{X}_{\text {MAX }}$}

By its very nature, the largest event in the dataset is a property of a dataset under consideration. For this reason I prefer the convention employed by Gibowicz and Kijko (1994), and Kijko and Funk (1994), who refer to this value as $X_{\max }$ (the maximum value of set $X$ ). 
The value of $X_{\max }$ is an indicator of hazard level in that it provides a lower bound of the largest event that can be expected in future $\left(M_{U L}>X_{\max }\right)$. Unless there is a significant and proven change in the conditions, the only defensible assumption is that the next largest event will be larger than $X_{\max }$. A significant change in the seismic regime can be brought about by, for example, a change in the mining method or when mining moves from strong brittle ground into squeezing conditions.

$X_{\max }$ is, of course, highly dependent on the size and representativeness of the subset of data under consideration and $X_{\max }$ of the subset loses significance as a hazard indicator when a subset is temporally or spatially too small. $X_{\max }$ as a hazard indicator captures only historical experience and does not in any way account for the stochastic nature of seismicity.

\section{THE a/b VALUE}

The value of a fitted GR relationship at $N=1$ is sometimes also referred to as ' $a / b$ ' (Hudyma, 2008), since it is equivalent to the ratio of the $a$ and $b$ parameters of the GR relationship (Equation 1). It is important to note that $a / b$ is a property of the fitted statistical model and not of the underlying data. The $a / b$ value is commonly used as an indicator of seismic hazard level with the meaning of 'the largest expected event' assigned to it.

It appears that this practice of interpreting $a / b$ as the maximum event size is reinforced by the misunderstanding of the frequency-magnitude graph plotted for historical data (Figure 2). As the minimum value of the logarithmic $y$-axis is generally plotted as 1 , and since no fractions of events can occur, this is interpreted as the 'end of the graph'. The GR relationship, however, is not a representation of the discrete events that occurred but a statistical best-fit model describing the relative frequency/probability of different event sizes. There is no fundamental reason to stop the graph at $N=1$. Interpreting the reverse cumulative distribution to terminate at $N=1$ ignores the upper tail of the distribution, similar to the red line illustrated in Figure 3.
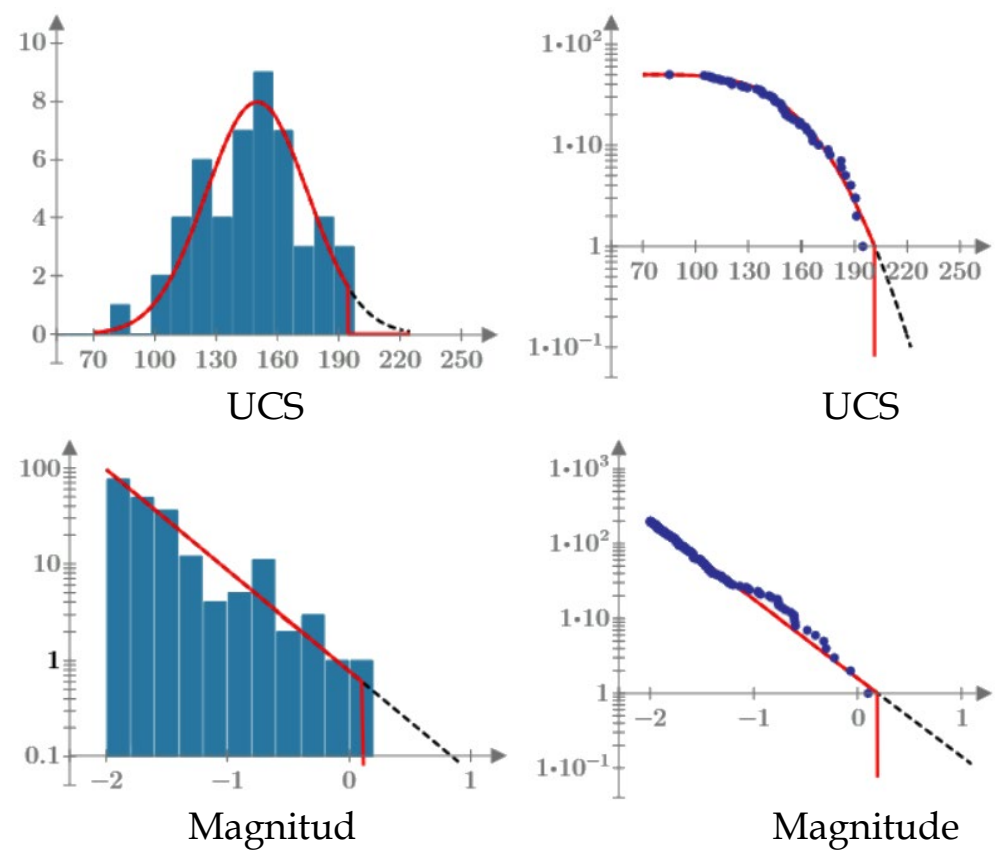

a) Histogram

b) Reverse Cumulative distribution

Figure 3. Truncation of statistical at $N=1$ to the largest point in the dataset for both UCS data and seismic magnitude data 
From the TGR distribution with the number of events determined by $a$, the probability of exceeding $a / b$ is given by the following equation derived in Appendix A and plotted in Figure 4:

$$
P\left[X_{\max } \geq \frac{a}{b}\right]=1-\left(\frac{1-10^{-a}}{1-10^{-a \cdot f}}\right)^{10^{a}}
$$

Which, for the open-ended GR relationship, reduces to the following:

$$
P\left[X_{\max } \geq \frac{a}{b}\right]=1-\left(1-10^{-a}\right)^{10^{a}}
$$

where:

$\begin{array}{ll}X_{\max } & =\text { the largest experienced event } \\ b & =\text { coefficient quantifying the log relative occurrence of smaller to larger events } \\ a & =\text { coefficient quantifying the number of events } N @ 0=10^{a} \\ f & =\text { Fraction by which } M_{u L} \text { is larger than } a / b, \text { i.e. } M_{U L}=f \cdot a / b\end{array}$

For an open-ended GR relationship, there is a $>63 \%$ chance of exceeding $a / b$. The probability is less for the truncated distribution. For situations where $M_{u L}$ is large compared to $a / b$, the probability of exceeding $a / b$ is large and the probability of exceeding $a / b$ reduces for $a / b$ closer to $M_{U L}$. The probability of exceeding $a / b$ where $a / b \geq M_{U L}$ is, per definition, zero. Note that these statements refer to the probability of exceeding $a / b$ within the number of events determined by the $a$, i.e $\mathrm{N} @ 0=10^{\text {a }}$.

The $a / b$-value is an important parameter and it has a very clear meaning not generally appreciated. The $a / b$-value is the mode of the distribution of the largest event (the mode of $f_{\max }$ ) (Figure 5). This is true for both the GR and TGR relationships (Appendix A).

The $a / b$ value is often used without consideration of spatial or temporal normalisation, in which case it is a function of the subset of data under consideration and it loses any meaning as a hazard indicator. This will be discussed in greater detail further in the paper.

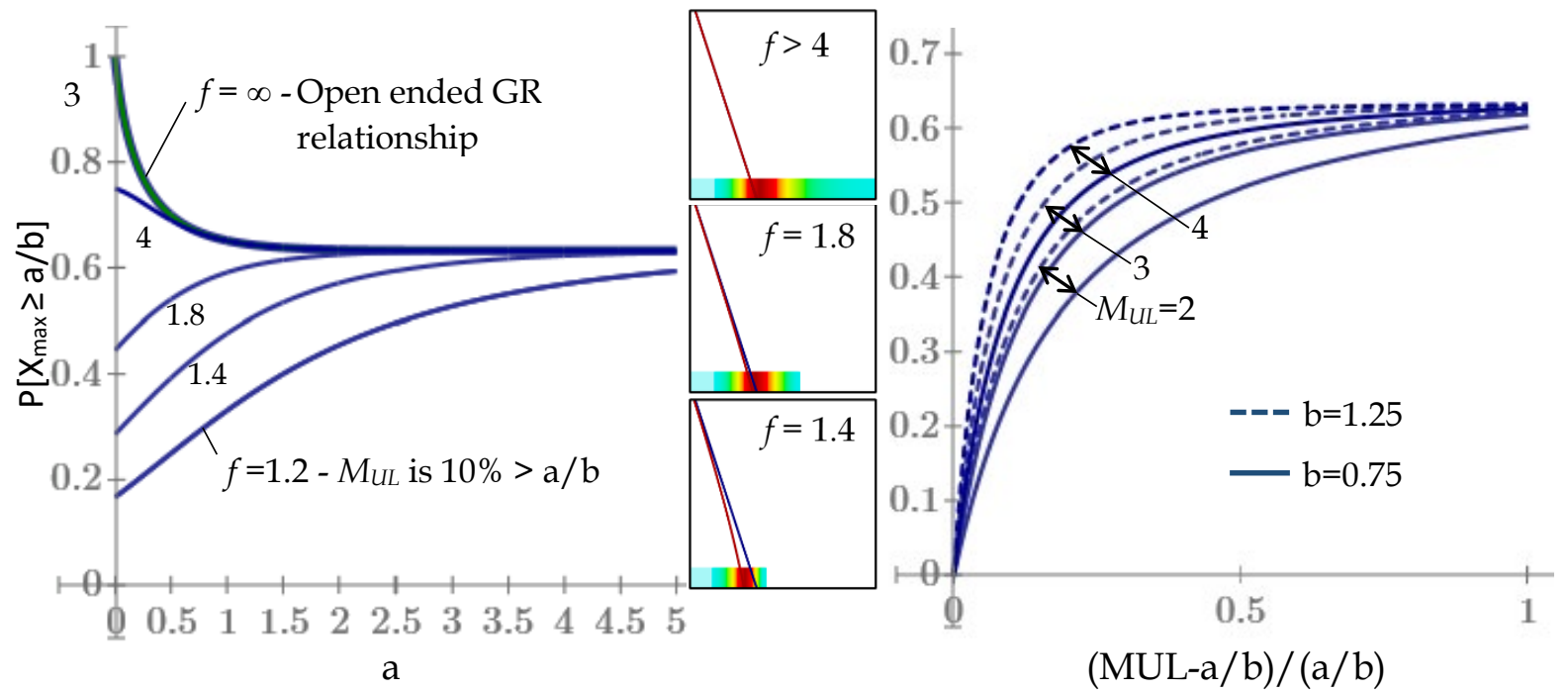

Figure 4. Probability for exceeding $a / b$ 


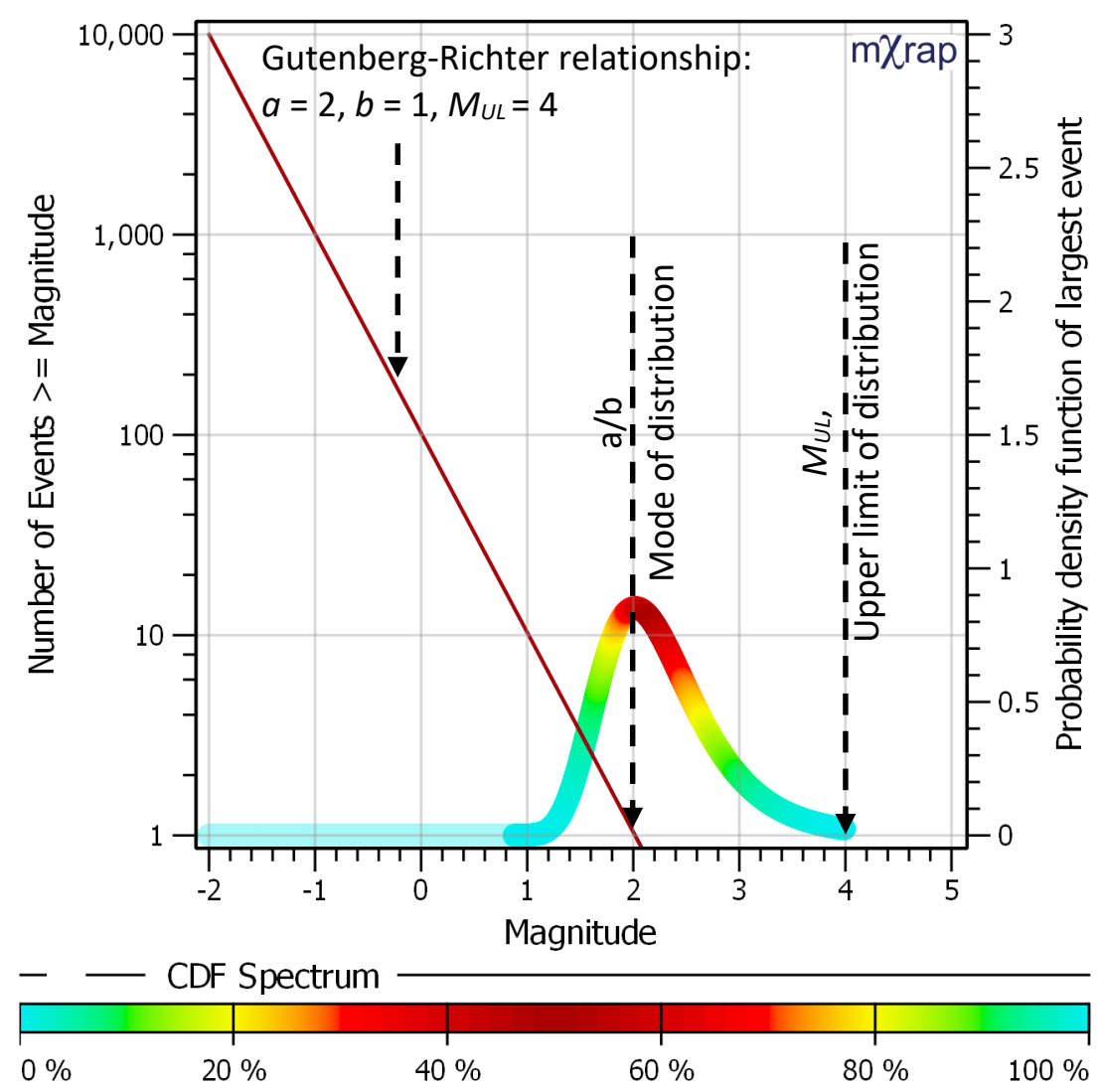

Figure 5. The a/b value as the mode of distribution of the largest events within a given number of events

\section{$M_{M A X}$ AND $M_{u L}$}

In physical terms, $M_{\max }$ is used to define the region-characteristic maximum possible event magnitude, or, as the upper limit of event magnitude for a given region (Kijko and Singh, 2011). In other words, the largest magnitude that physical conditions will allow. In crustal seismology, this value is generally assumed to be constant for a particular seismic source zone. However, in the mining environment, the value of $M_{\max }$ is not constant and is influenced by a number of factors, for example: rock mass conditions, mining-induced stress state, the mining sequence, and mining layout. In addition, $M_{\max }$ is expected to increase with the extraction ratio (Mendecki, 2012).

As mentioned before $M_{\max }$ and $M_{U L}$, are mostly used interchangeably. Conceptually $M_{\max }$ has the previously mentioned physical meaning, whilst $M_{u L}$ carries only the abstract meaning of being an upper limit of a probability distribution. I find it useful to keep these two concepts separate as one may choose to use a large value for $M_{U L}$, say M6, without implying that $M_{\max }=6$. The only implicit statement is that $M_{\max }<6$. Due to several difficulties which will be discussed in further detail in following paragraphs, estimates of $M_{\max }$ is subject to a great deal of uncertainty. For the purpose of hazard assessment and management, however, choosing a conservative but realistic value for $M_{U L}$, will suffice.

When assessing the probability of exceeding a specified magnitude $P\left[M>M_{L}\right]$, underestimation of $M_{u L}$ with and error of $\delta\left(M_{U L}=M_{\max }-\delta\right)$ leads to larger errors than overestimation of $M_{U L}$ by the same amount $\left(M_{U L}=M_{\max }+\delta\right)$. Underestimating $M_{U L}$ is always optimistic, whilst overestimation is always conservative (Wesseloo, 2018). For the purpose of hazard assessment, it is, therefore, prudent to use values for $M_{u L}$ that are deliberately conservative. 
Estimating $M_{\max }$ and $M_{u L}$

Wesseloo (2018) suggested the use of several methods discussed by Kijko (2004) to calculate $M_{\max }$ plus the associated standard deviation, and assigning the maximum of these values to $M_{u L}$.

$$
M_{U L}=\max \left(M_{\max i}+\Delta_{i}\right)
$$

Where:

$M_{u L}=$ conservative but realistic upper limit for $f_{\max }$.

$M_{\max }=\quad$ the largest magnitude that physical conditions will allow estimated with method $i$.

$\Delta_{i} \quad=\quad$ standard deviation of the estimation of $M_{\max i}$

The reliable and robust estimate of $M_{\max }$ is not a trivial task and several researchers have invested considerable effort into finding reliable estimates from seismic event catalogues, with most of the effort directed at application in crustal seismology (e.g. Kijko and Funk, 1994, Kijko, 2004, Lasocki and Urban, 2011, Kijko, 2012). These methods, based on record statistics, aim to estimate the largest possible value based on the recorded data.

The simplest and most well-known method for estimation of $M_{U L}$ is the Robson-Whitlock method formulated as follows (Kijko, 2004):

$$
M_{\max }=X_{\max }+\left(X_{\max }-X_{\max -1}\right)
$$

Where

$X_{\max }$ and $X_{\max -1}=\quad$ is the largest and second largest recorded events

Among the other more common methods discussed by Kijko (2004) and (Kijko and Singh, 2011) are the Tate-Pisarenko, Kijko-Sellevoll, Order statistics, and Robson-Whitlock-Cooke and Cooke 1980.

It is important to note that the reliability of the estimate of $M_{\max }$ is highly dependent on the number of events on which this assessment is based. This dependence is investigated using Monte Carlo analysis on synthetically generated datasets sampled from a specified FM distribution. For this analysis, $M_{\max }=2.5,3.5$ and $4.5, b=0.75,1$ and 1.25 , and $a$-values from 1 to $4.5(\mathrm{~N} @ 0=10$ to 32000$)$ were used.

The results for the Robson-Whitlock method is shown in Figure 6 or $b$-values of 1 . The results of this analysis are combined in a normalised chart in Figure 7a: normalised for all $M_{\max }, a$ - and $b$-values. For $a / b \ll M_{\max }$, the accuracy of the estimate is very low. In the analysis performed, the value for $M_{\max }$ is largely underestimated, with an underestimation of more $80 \%$ for an $a / b<M_{\max }-1$. Similar results were obtained when performing the same analysis and taking $M_{\max }$, the maximum obtained from the TatePisarenko, Kijko-Sellevoll, Order statistics, Robson-Whitlock-Cooke and Cooke 1980 methods Figure $7 \mathrm{~b}$.

It is clear from this analysis that reasonably accurate estimates for $M_{\max }$ can only be obtained from large datasets. Sometimes $M_{\max }$ is estimated for small subsets of data, a practice that should be avoided. Reliable results can only be obtained when $M_{\max }$ is based on large datasets ( $\mathrm{N} @ 0>1000$ for $M_{\max }=2.5$ $\mathrm{N} @ 0>10000)$ for $M_{\max }=4.5$. 

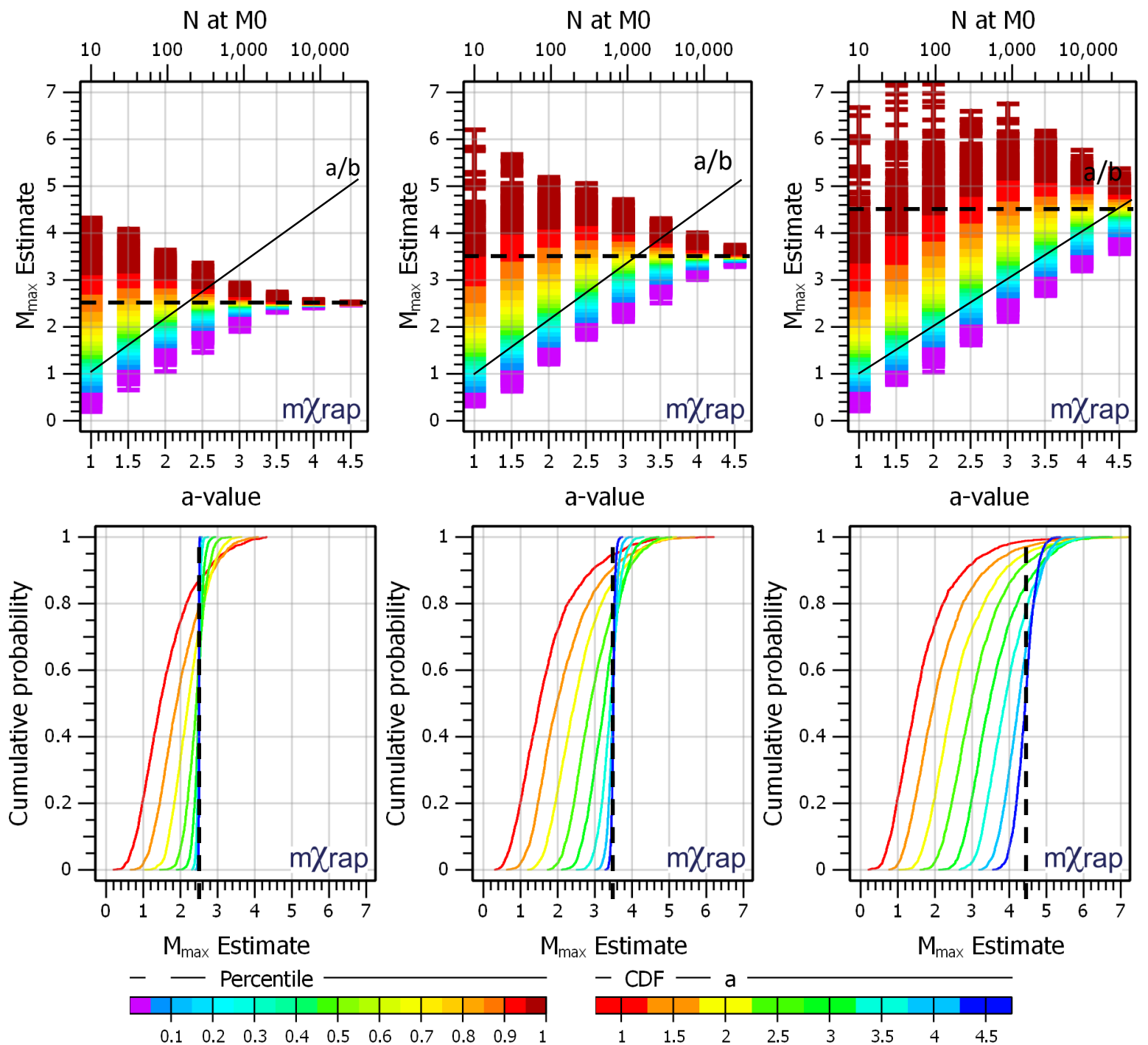
a) True $M_{\max }=2.5$
b) True $M_{\max }=3.5$
c) True $M_{\max }=4.5$

Figure 6. Uncertainty in the estimation of $M_{\max }$ using the Robson-Whitlock method, $b=1$ 

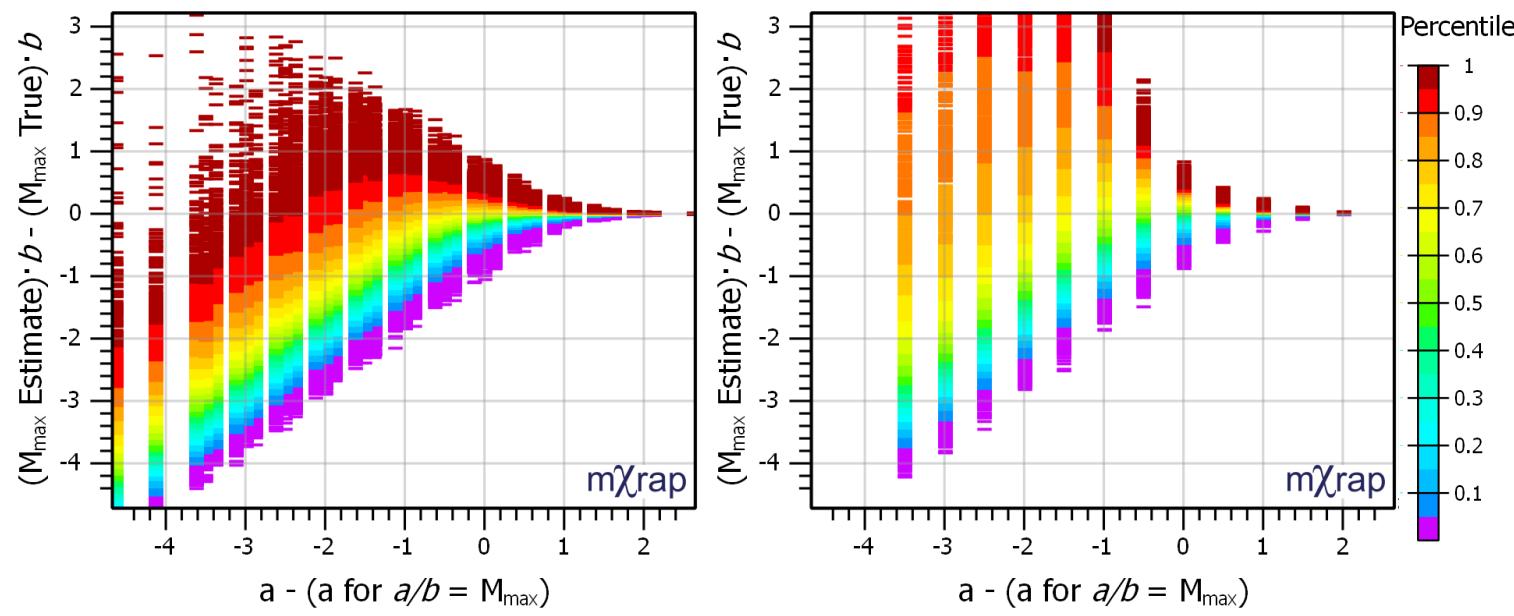

Figure 7. Uncertainty in the estimation of $M_{\max }$ for a) Robson-Whitlock b) using the maximum value obtained from Tate-Pisarenko, Kijko-Sellevoll, Order statistics, Robson-Whitlock-Cooke and Cooke 1980 (Kijko, 2004). Normalised for all values of $M_{\max }, b$ and $a$

\section{Artificial Black Swan Events}

\section{The effect of variability}

According to the Financial Times Lexicon, a Black Swan Event is "An event or occurrence that deviates beyond what is normally expected of a situation and that would be extremely difficult to predict." Since the $M_{U L}$ is the upper asymptote of the FM distribution, any event with magnitude greater than $M_{U L}$ is, per definition, assigned a zero probability of occurrence. Underestimation of $M_{U L}\left(M_{U L}<\right.$ the true $\left.M_{\max }\right)$, will assign a zero probability to magnitudes that could reasonably be expected, should the $M_{U L}>$ the true value of $M_{\max }$.

Consider the following example based on synthetic magnitude distribution data. Using random deviate sampling, magnitude values were sampled from a TGR relationship with the following properties: $M_{U L}=4, m_{\min }=-2, b=1$ and N@-2 is 100000 , i.e. $a=3$. The results of several different approaches for estimating $M_{U L}$ are shown in Figure 8. 

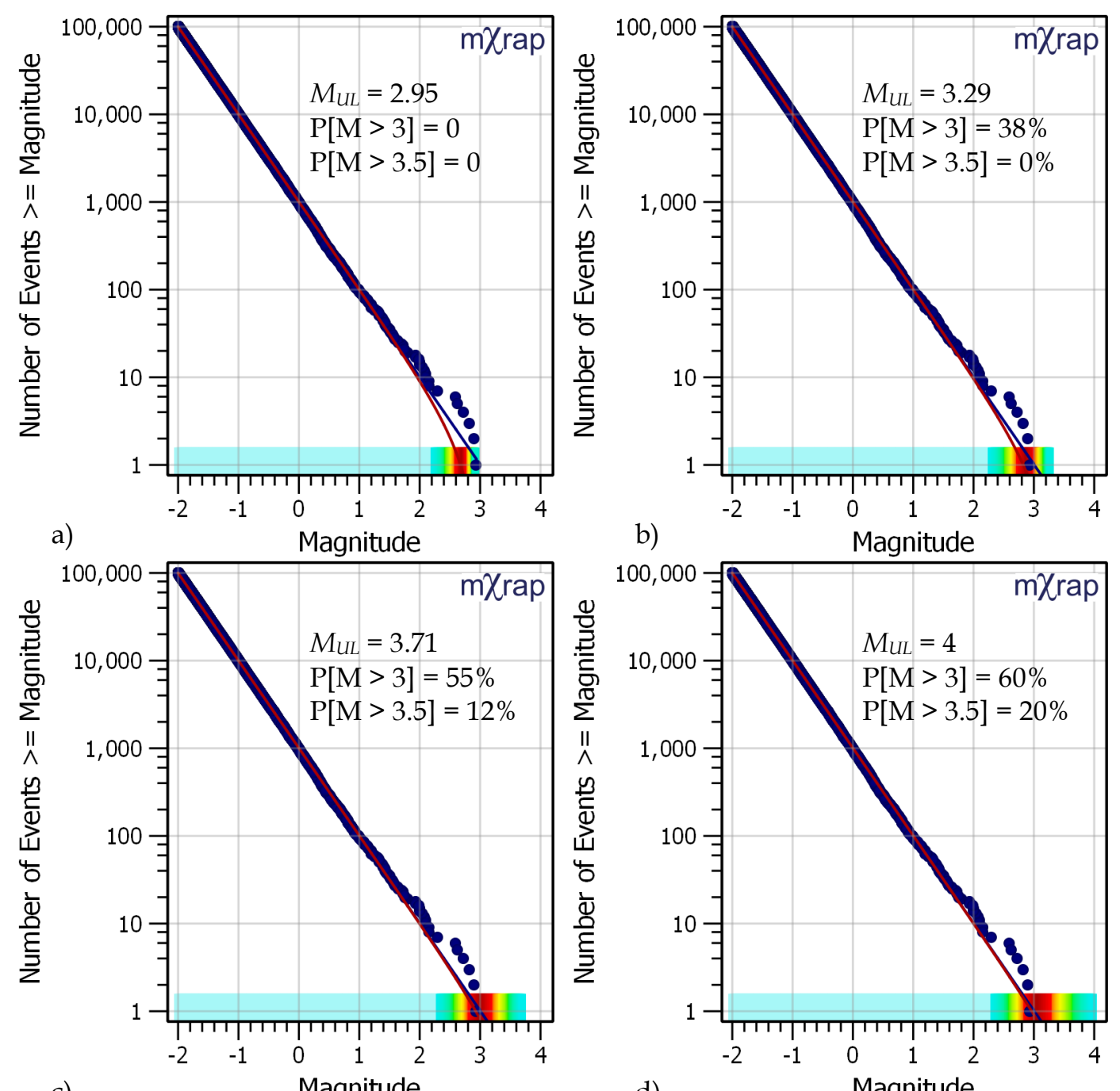

b)

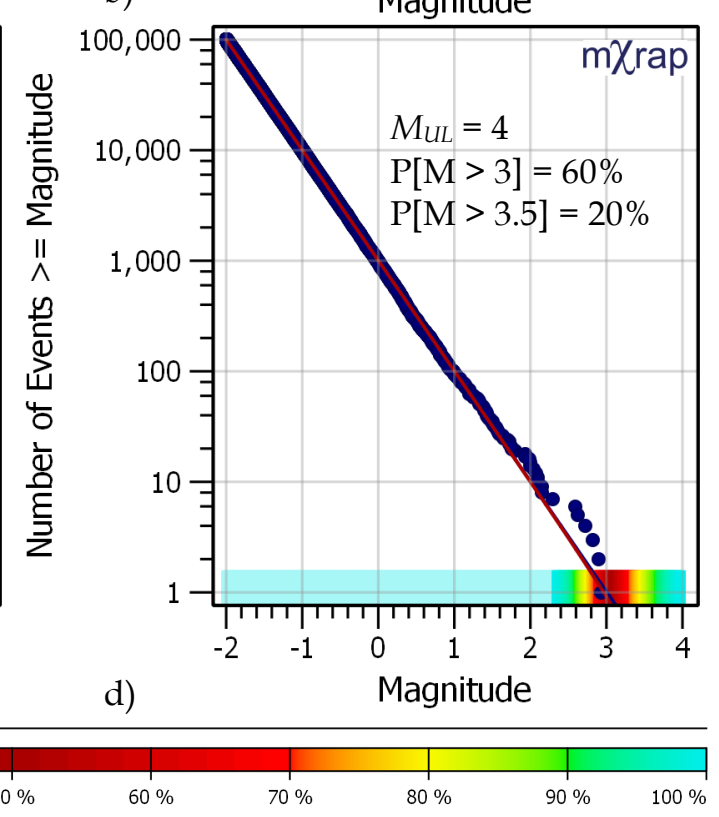

$$
\begin{aligned}
& -\mathrm{Ma} \\
& \hline 0 \%
\end{aligned}
$$$$
\operatorname{Max}(\mathrm{M})
$$$$
\text { Magnitude }
$$$$
10 \% \quad 20 \% \quad 30 \% \quad 40 \% \quad 50 \%
$$

Figure 8. Comparison to probabilistic evaluations resulting from different values of $M_{U L}$

Each of the sub-figures show the FM distribution data; the best fit GR (blue) and TGR (red) relationships. The probability distribution of the largest event for the given TGR relationship is shown as a spectrum at $N=1$. The only differences between the sub-figures are the $M_{U L}$ value and the resulting changes in the TGR relationship and the distribution of largest events, $f_{\max }$, derived from it.

In Figure 8a), the value of $M_{u L}=M_{\max }$ is obtained with the Robson-Whitlock method (Equation 12). In Figure $8 \mathrm{~b}), M_{U L}$ is taken as the maximum of all of the methods listed in the previous section. In Figure 8c), the $M_{u L}$ is taken as the maximum of all the aforementioned methods with an added standard deviation for each calculation, according to Kijko and Singh (2011), assuming a magnitude resolution of 0.1. Finally, in Figure 8d), $M_{u L}=$ the true $M_{\max }=4$ is used. Even with $M_{u L}$ calculated, as suggested in Equation 11, (Figure $8 b$ ), the probability of $M>3.5$ is zero whilst the actual value is $20 \%$.

\section{The effect of sensor limitations}

Apart from the underestimation of $M_{\max }$ that may occur simply due to variability in the data, underestimation may also result from limitations in lower frequency range of sensors. Morkel and Wesseloo (2017) described the problem occurring when the lower frequency limit of the sensors are not 
sufficiently low to record the low frequency content of large events. When this occurs, it will lead to the under-recording of the moment (and potency) and magnitude. As a result, the FM distribution of the recorded data exhibits a non-linear (on the log-linear scale) relationship, as shown in Figure 9.

Figure 9a shows the FM distribution of recorded data from a mine network with only $50 \mathrm{~Hz}$ sensors. The downward curvature of the distribution is not a result of the underlying statistical behaviour of magnitude, but of the under-recording of the moment by the sensors. Also shown in the figure are two theoretical lines: the straight GR relationship assumed as the true distribution of magnitude; and a theoretical assessment of the effect of under-recording using analytical formulations (Boore, 1986, Di Bona and Rovelli, 1988, Mendecki, 2013, Morkel and Wesseloo, 2017). The effect of different lower frequency limits of the sensors is illustrated in Figure 9b. The amount of under-recording for different magnitudes and lower frequency limits are shown in Figure 10.

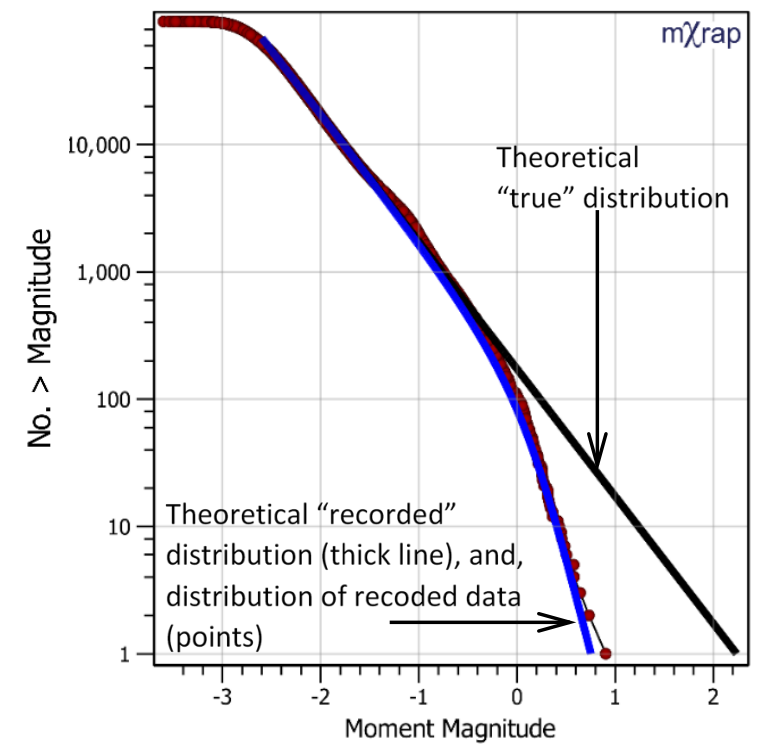

a) Database and theoretical estimation of a $50 \mathrm{~Hz}$ system.

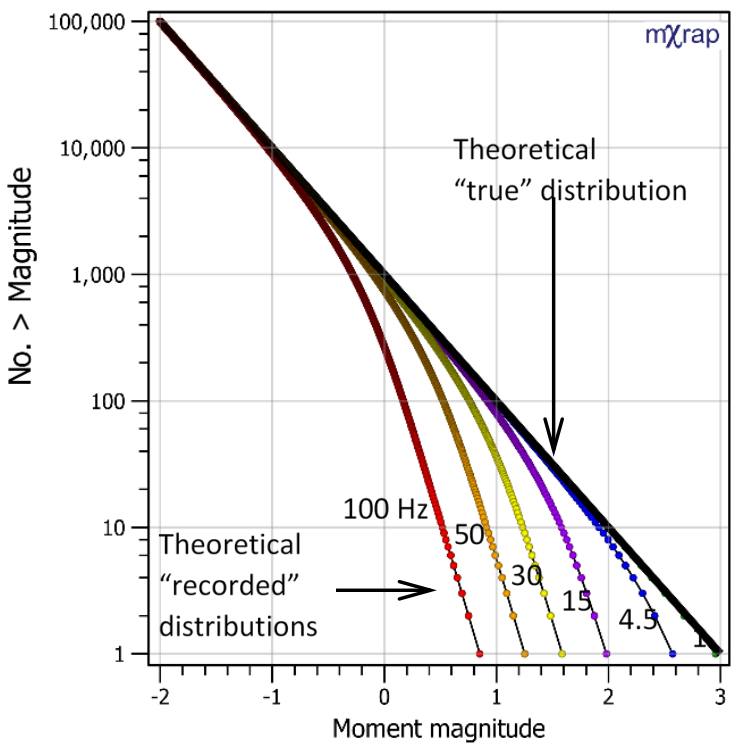

b) Theoretical estimation of moment magnitude under-recording for different lower frequency limits

Figure 9. The effect of sensor frequency limits on the FM distribution of recorded seismic datasets (Morkel and Wesseloo, 2017) 


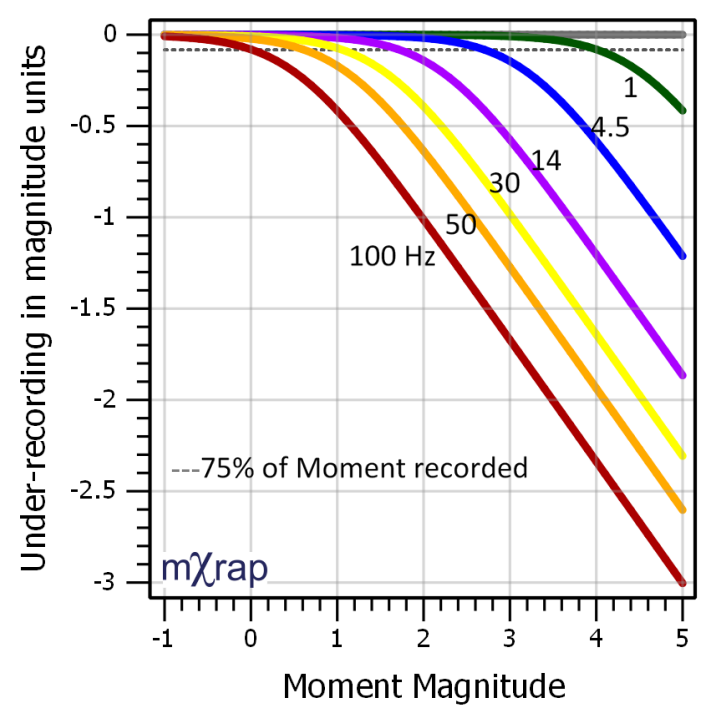

a) Static stress drop $=0.1 \mathrm{MPa}$

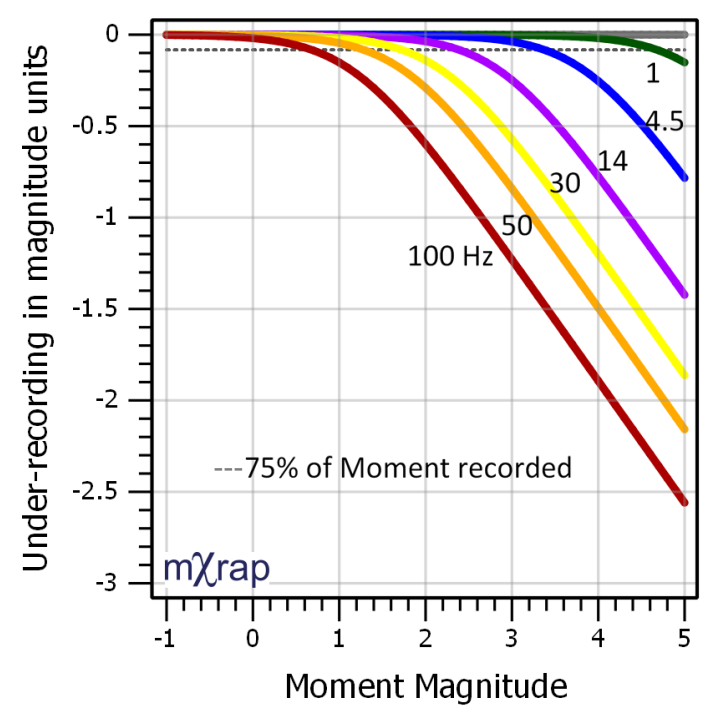

b) Static stress drop $=1 \mathrm{MPa}$

Figure 10. Under-recording of Moment magnitude for different magnitudes and lower frequency limits For databases subjected to under-recording, optimistic values of $M_{\max }$ will result from the use of the statistical methods. To illustrate this problem, consider the following scenario plotted for $\mathrm{N} @ 0=300$ in Figure 11. A seismic system with sensor lower limits of $14 \mathrm{~Hz}$ recording events from a seismic source with a $b=1, M_{\max }=3$. Figure 11 shows the assumed FM distribution as a blue line, and the synthetic events sampled from that distribution as light grey. The dark blue points are adjusted for the frequency limit of the sensors, according to Boore (1986). The value of $M_{\max }$ in this case is 2.53 which is smaller than the value of the largest experienced event on which the assessment is based M2.68. The red line shows the truncated GR relationship based on the recorded events. Similar responses also occur for other frequency limits, but the magnitude range at which the deviation becomes significant, differs. The lower the frequency limit of the sensor, the larger the magnitude that will be adequately recorded.

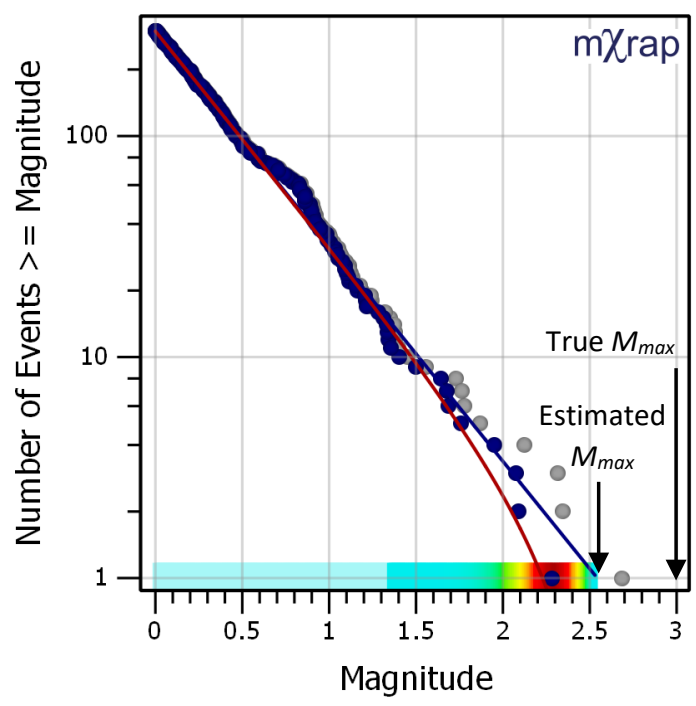

Figure 11. Synthetic data with adjustments for frequency under-recording lower frequency $=14 \mathrm{~Hz}$ and a static stress drop of $0.1 \mathrm{MPa}$

To further quantify the effect of the lower frequency of sensors on the estimation of $M_{\max }$, a Monte Carlo analysis was performed, similar to that for which the results are shown in Figure 6 and Figure 7. For this analysis, we assume a lower frequency limit of $14 \mathrm{~Hz}$ and a static stress drop of $1 \mathrm{MPa}$. Excluding the added effect of the lower frequency of the sensor, these two analyses are the same. The results of 
this analysis are shown in Figure 12. It is clear from comparison between Figure 6 and Figure 12 that the under-recording of moment due to sensor limitation can result in errors in the estimation of $M_{\max }$ which is always optimistic and can be significant.

The best solution to this problem is to include lower frequency sensors in the system which are able to adequately record the lower frequency content of the event sizes expected at the mine (Figure 10). In lieu of this, corrections may be applied to the recorded values to compensate for the effect of the sensor under-recording (Morkel and Wesseloo, 2017).
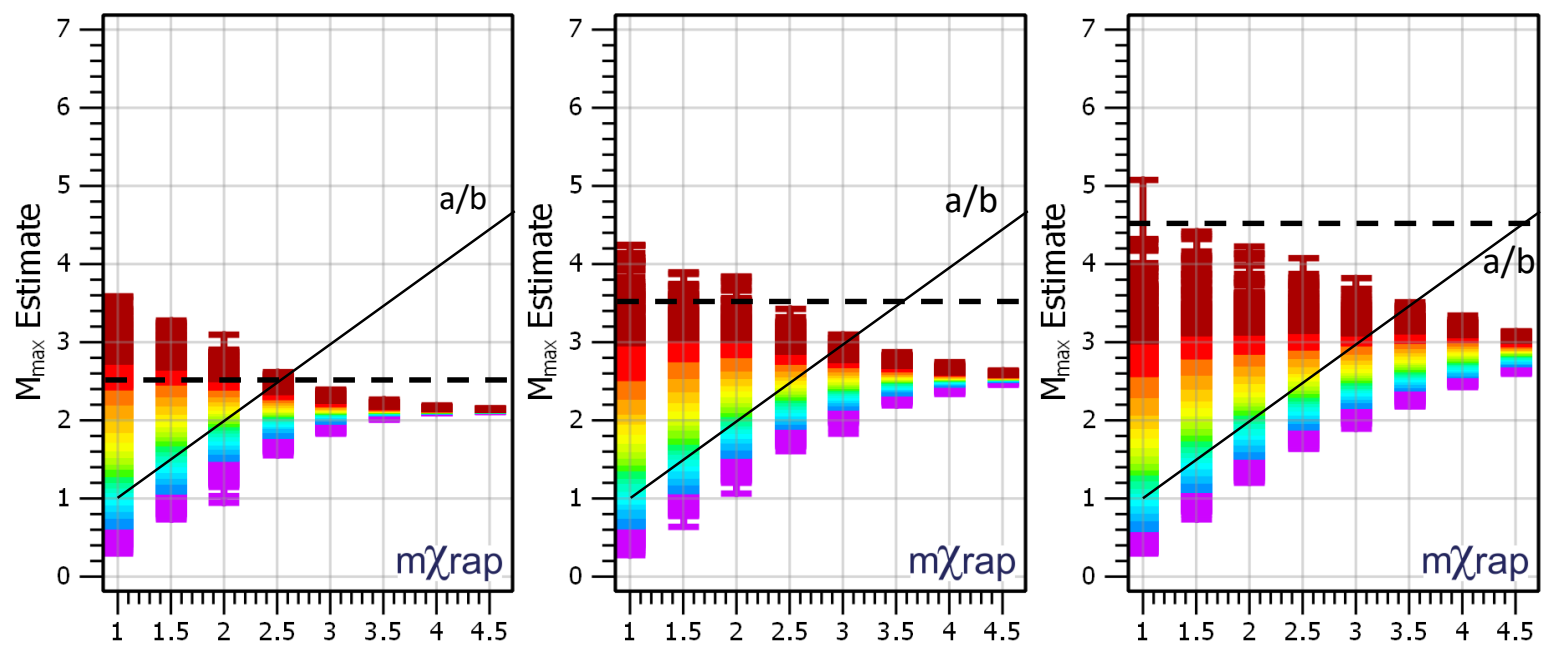

a-value $\left(\mathrm{N}\right.$ at $\left.\mathrm{MO}=10^{\mathrm{a}}\right)$
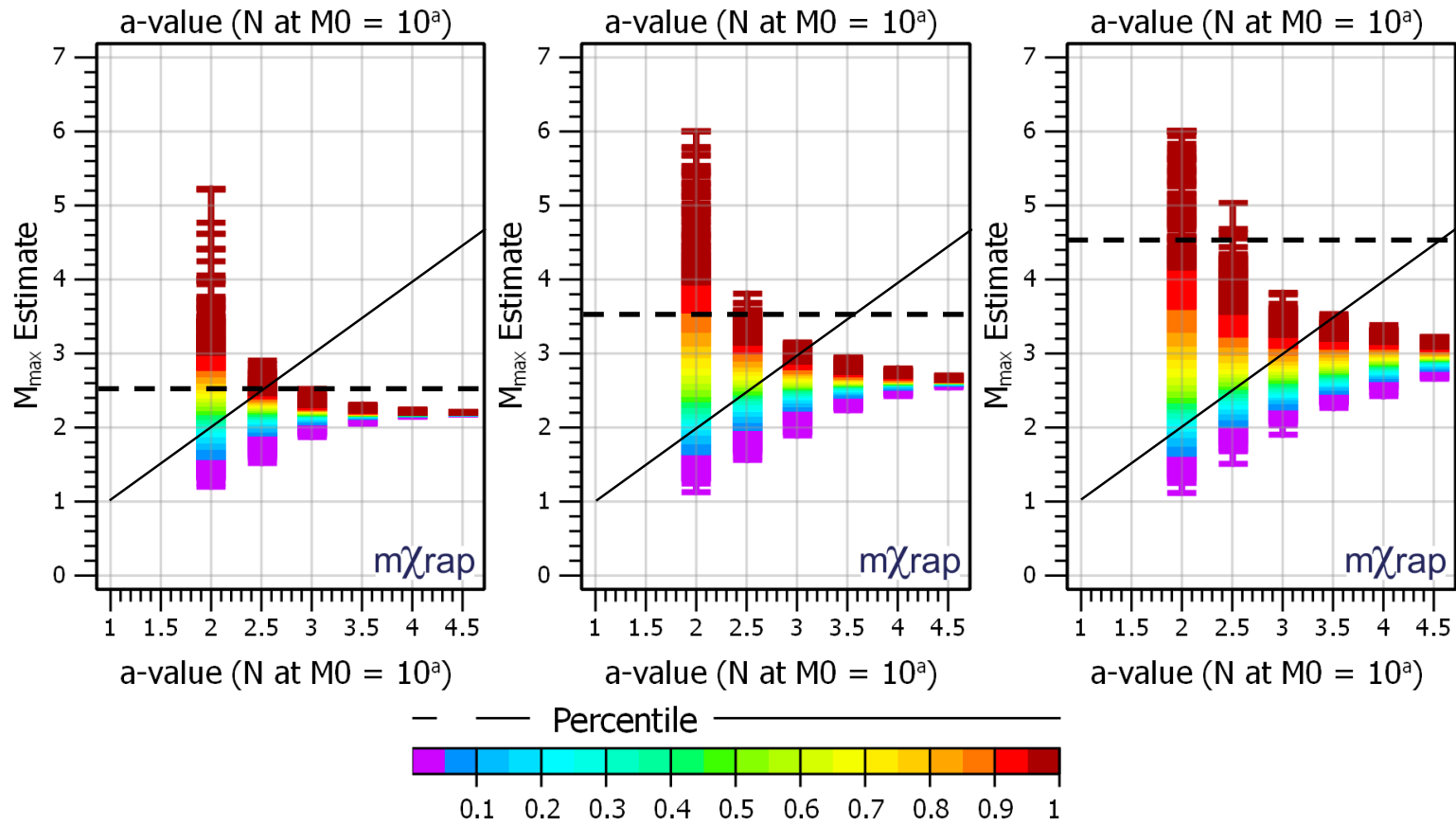
a) True $M_{\max }=2.5$
b) True $M_{\max }=3.5$
c)
True $M_{\max }=4.5$

Figure 12. Uncertainty in the estimation of $M_{\max }$ using the Tate-Pisarenko, Kijko-Sellevoll, Order statistics, Robson-Whitlock-Cooke and Cooke 1980 methods. Lower frequency $=14 \mathrm{~Hz}, b=1$, Static stress drop $=1 \mathrm{MPa}$ 


\section{SPATIAL DISTRIBUTION OF SEISMIC HAZARD}

Probabilistic hazard calculation is commonly performed on data within spatial filters. Such spatial filters are often delineated with respect to mining infrastructure, often with arbitrary size. Such arbitrarily chosen volumes can have a significant influence on the assessment and may influence decision-making.

The sensitivity of hazard assessment to arbitrarily chosen volumes relates to the spatial distribution of $b$-value, the spatial distribution of events and the difference in volume for these arbitrary spatial filters.

\section{The influence of the spatial distribution of $b$-value}

It is important to note that, when different seismic sources with different $b$-values are lumped together and the hazard calculated, the total hazard will be different than calculating the total hazard based on the separate sources.

To illustrate this point, consider the idealised case illustrated in Figure 13 with a square subdivided into equal sub-areas on the left-hand side. On the right-hand side, the whole area is evaluated as a single unit. By way of analogy, these squares represent a mining area. For this illustration, we would like to answer the following question:

What is the probability of $M \geq 2$ ?

For the purpose of this illustration we will assume a constant event rate.

An approach often used in mining is to evaluate the whole area, $A$, obtaining the $a$ and $b$-values for the whole area, and calculate the required probability value using the following equation.

$$
P(M \geq M L)_{A}=1-F_{\max }\left(a_{A}, b_{A}, M L\right)
$$

An alternative approach would be to calculate the $a$ and $b$-values for each of the sub-areas and calculate the overall probability as follows:

$$
\begin{aligned}
& P(M \geq M L)_{i}=1-F_{\max }\left(a_{i}, b_{i}, M L\right) \\
& P(M \geq M L)_{A}=1-\prod_{i} 1-P(M \geq M L)_{i}
\end{aligned}
$$

The $b$-value for the combined area can be obtained from that of the sub-volumes as follows:

$$
b=\frac{\sum_{i} 10^{a_{i}}}{\sum_{i} \frac{1 a_{i}}{b_{i}}}
$$

Where:

$a_{i}, b_{i}=a$ and $b$ values for sub-volume $i$

It can be shown that for cases where the $b$-value over area $A$ is constant, i.e. where $b_{\mathrm{i}}=b_{\mathrm{A}}$, the two approaches yield the same result. However, where this is not the case, the first approach does not yield the correct answer. For the example, in Figure 13 the difference between the two approaches leads to a difference of $9 \%$ probability of exceeding M2. 


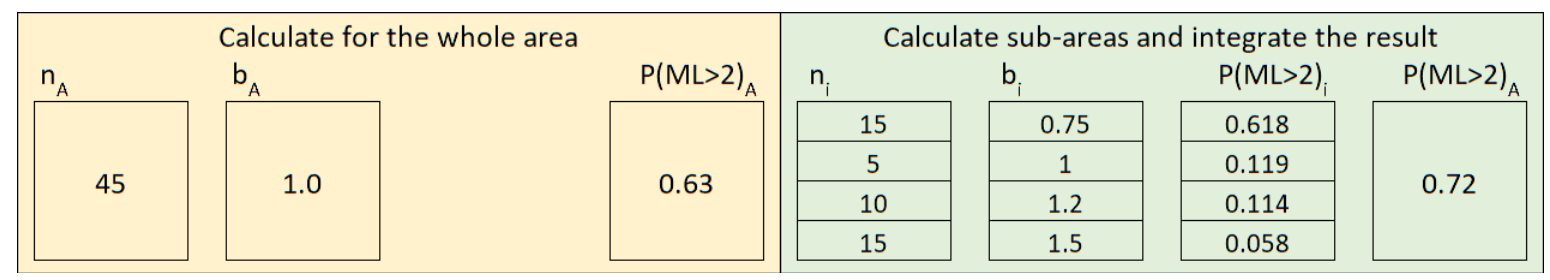

Figure 13. Illustration of the difference between evaluating the seismic hazard as a single unit and for smaller units with constant b-value

This illustration shows that the probabilistic evaluation representative of the volume under consideration can only be achieved by integration of the results obtained for each sub-volume where the sub-volumes are small enough to represent a volume with constant $b$-value.

\section{The influence of the volume of spatial filters}

For a given $b$ - and $M_{U L}$ value, the number of events within a spatial filter will determine the seismic hazard for that spatial filter. The effect of hazard quantification and comparison between arbitrarily chosen spatial filter volumes without volume normalisation is illustrated in Figure 14 and Figure 16. The square area shown on the left-hand side in Figure 14 and Figure 16 represents a whole mining area. In the left-hand side figures, the whole area consists of a hundred equal sized sub-areas which, for this example, each has a uniform distribution of events. The right-hand side figures display the same scenarios as that of the left-hand side figures, except that in these cases the whole mining area is subdivided into arbitrarily chosen spatial filter areas.

In Figure 14, the whole mine has the same seismic event rate of $\mathrm{N} @ 0=10$ and $b=1$. As a result, the spatial distribution of hazard is uniform throughout the whole mine with an $a / b=1$ (Figure 14a left), and a probability of exceeding M2.5 at $3 \%$ per small square (Figure $14 \mathrm{~b}$ left). The $a / b$ values and the probability of exceeding M2.5 is shown in the right-hand side figure for each of the arbitrary spatial filter volumes. The highlighted sub-area consists of 42 small areas and therefore has $\mathrm{N} @ 0=420$. The TGR (red line), $a / b, f_{\max }$ (coloured distribution) for a small square and the highlighted area are shown in Figure 15.

Evaluating the seismic hazard for arbitrary volume sizes leads to the amplification of the hazard for larger volumes and a misrepresentation of the hazard. Figure 16 illustrates the same effect in a different scenario where the event rate is not uniformly distributed throughout the whole mining area. Figure 16 illustrates the fact that arbitrarily chosen filter volumes can mask the true spatial distribution of seismic hazard. These comparative hazard maps can be corrected by normalising the assessment with respect to the volume. 


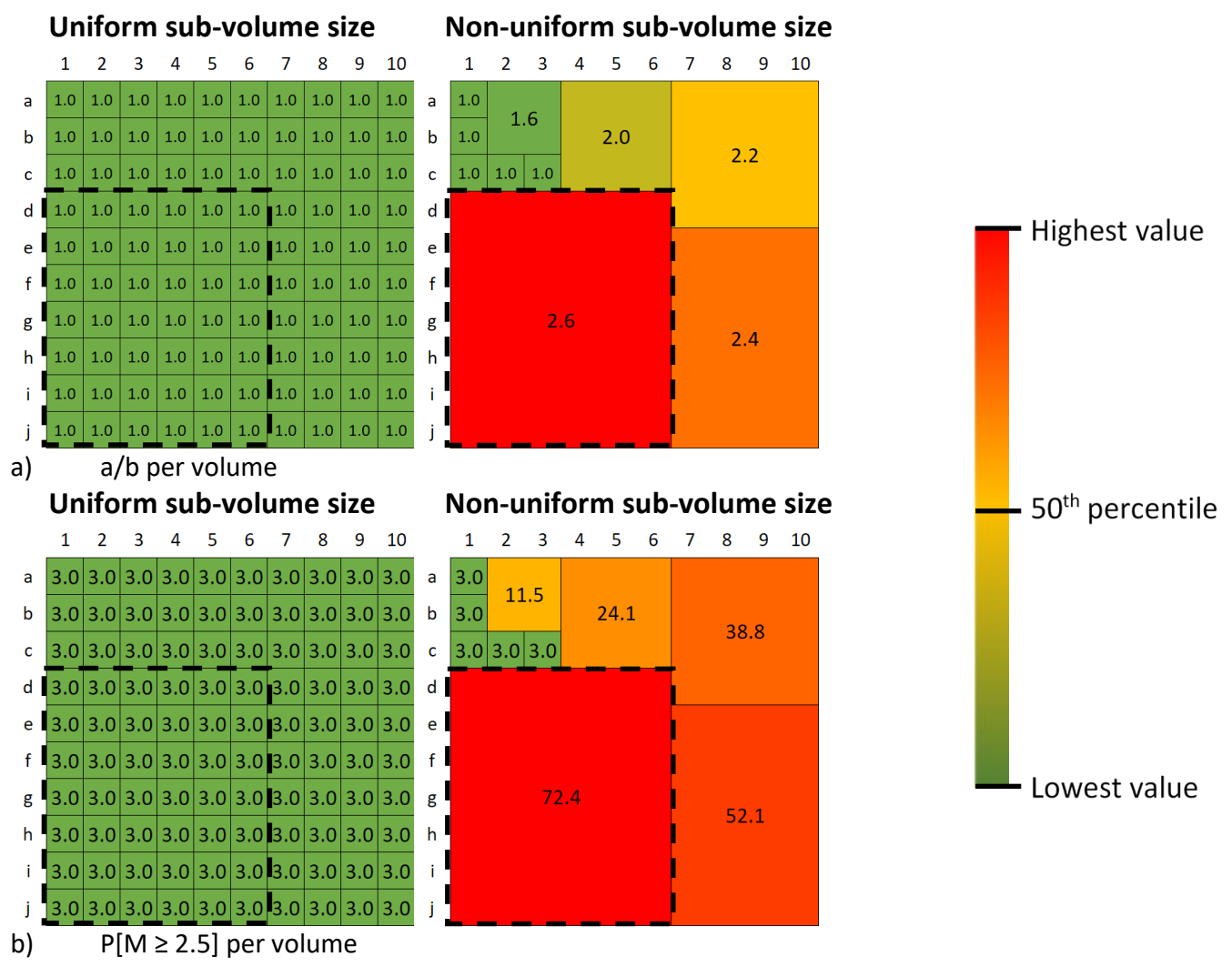

Figure 14. The influence of volume size on the evaluation of hazard a) a/b distribution for uniform volume size and b) probability of exceeding $M 2.5, M_{u L}=4, m_{\min }=0$

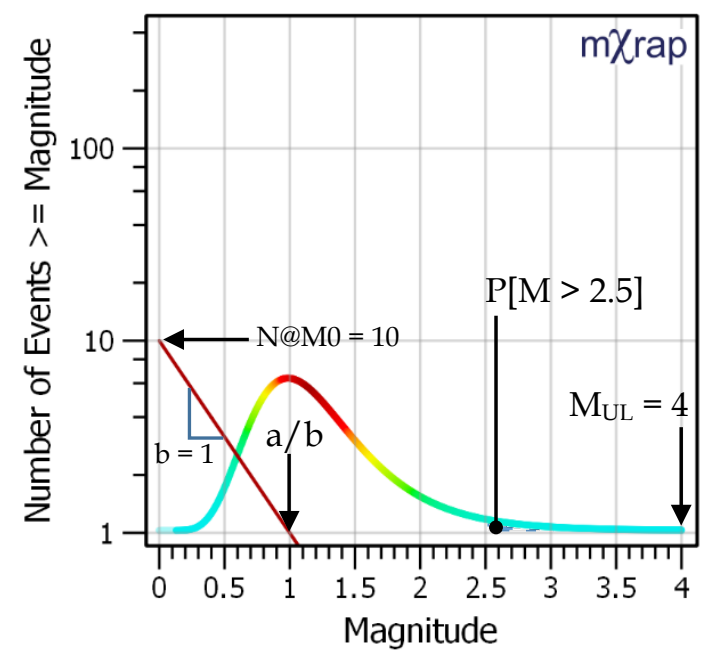

a) Small square

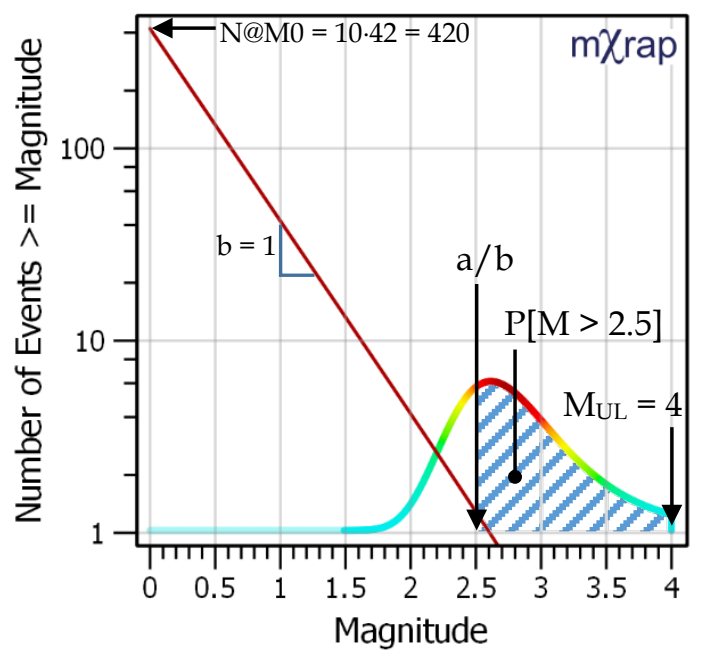

b) highlighted area

Figure 15. The TGR (red line), $a / b$, and $f_{\max }$ (coloured distribution) for the a) small squares, and $b)$ the highlighted area in Figure 14 

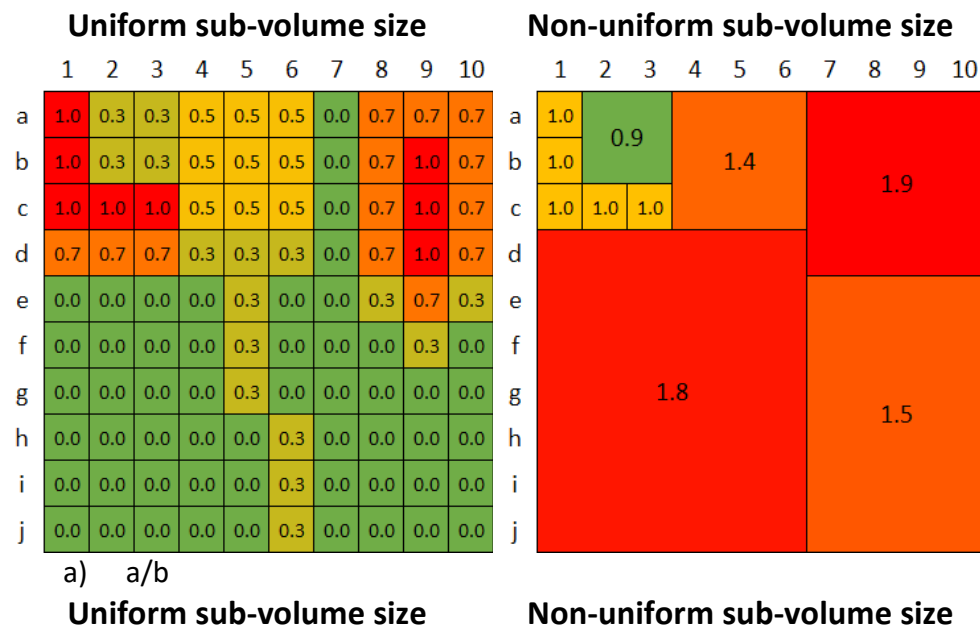

\section{Non-uniform sub-volume size}

$\begin{array}{llllllllll}1 & 2 & 3 & 4 & 5 & 6 & 7 & 8 & 9 & 10\end{array}$
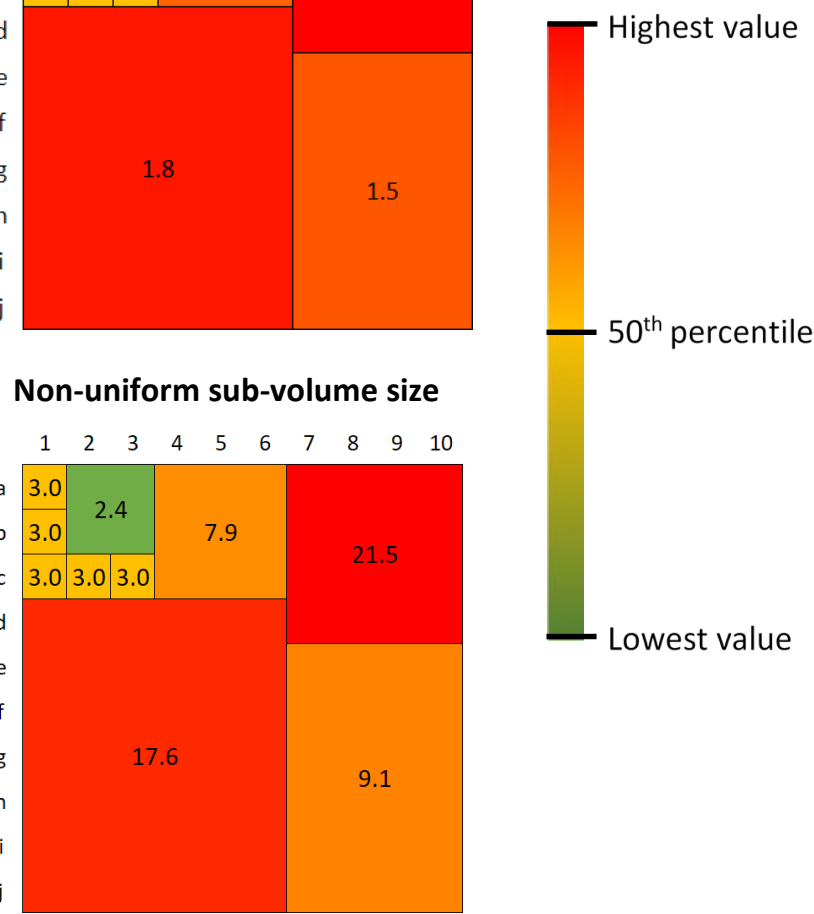

\begin{tabular}{lll|l|l|l|l|l|l|l|l}
1.5 & 1.5 & 1.5 & 0.6 & 0.6 & 0.6 & 0.3 & 1.5 & 3.0 & 1.5 \\
\hline
\end{tabular}

\begin{tabular}{lll|l|l|l|l|l|l|l}
0.3 & 0.3 & 0.3 & 0.3 & 0.6 & 0.3 & 0.3 & 0.6 & 1.5 & 0.6
\end{tabular}

\begin{tabular}{lll|l|l|l|l|l|l|l}
0.3 & 0.3 & 0.3 & 0.3 & 0.6 & 0.3 & 0.3 & 0.3 & 0.6 & 0.3
\end{tabular}

$\begin{array}{lllllllllll}0.3 & 0.3 & 0.3 & 0.3 & 0.6 & 0.3 & 0.3 & 0.3 & 0.3 & 0.3\end{array}$

\begin{tabular}{llllll|l|l|l|l}
0.3 & 0.3 & 0.3 & 0.3 & 0.3 & 0.6 & 0.3 & 0.3 & 0.3 & 0.3 \\
$h$
\end{tabular}

\begin{tabular}{lll|l|l|l|l|l|l|l}
0.3 & 0.3 & 0.3 & 0.3 & 0.3 & 0.6 & 0.3 & 0.3 & 0.3 & 0.3
\end{tabular}

\begin{tabular}{lll|l|l|l|l|l|l|l}
0.3 & 0.3 & 0.3 & 0.3 & 0.3 & 0.6 & 0.3 & 0.3 & 0.3 & 0.3
\end{tabular}

b) $\mathrm{P}[\mathrm{M} \geq 2.5]$

Figure 16. The influence of volume size on the evaluation of hazard a) $a / b$ distribution and b) probability of exceeding $M 2.5, M_{U L}=4, m_{\min }=0$

\section{HAZARD NORMALISATION}

\section{Spatial normalisation}

For the case illustrated in Figure 14, the statement was made that the probability of exceeding M2.5 per small square is at $3 \%$. In this statement, the hazard is normalised with respect to volume. This normalised value is essential for obtaining a spatial distribution of hazard or a true comparison of hazard between different volumes, but is does not provide the absolute hazard. The hazard for the whole mining area is not $3 \%$. For the whole mining area, we need to integrate the hazard of each of the sub-areas to obtain $95 \%$. In other words, there is about a 95\% chance of experiencing M2.5 anywhere in the mine, but it is equally likely to occur anywhere in the mine with a $3 \%$ probability of occurring in any one of the small squares. The total hazard for the whole mine and the spatial distribution of that hazard is independent of the size of the small squares, but the actual value associated with the small square, is dependent on the size of the squares. To represent the spatial distribution in a way that is independent of the subsquare size, one would need to define a characteristic volume to which all values are normalised. Wesseloo (2018) suggested the use of a volume size equal to that of a sphere with radius of $50 \mathrm{~m}$. The $a / b$ values in the scenario shown in Figure 16 is spatially normalised to a characteristic volume shown in Figure 17a.

The sub-square size of $20 \mathrm{~m}$ was assumed and since the example is a two-dimensional one, an equivalent representative area with radius $50 \mathrm{~m}$ was used. The comparison between the non-uniform sub-volume scenarios in Figure 16a and Figure 17a shows that the normalisation to a characteristic volume enables 
a more reasonable comparison of seismic hazard distribution. Spatial normalisation, however, does not correct for the loss of information that occurs when the spatial filters do not take account of the underlying seismic sources, as only a mean value for each sub-volume is retained. As a result, some masking of seismic hazard trends still occur. The left-hand side picture of Figure 17a illustrates the fact that when the evaluation volume is small enough to capture the change in seismic sources in space, the normalised $a / b$ values provide a useful hazard rating for quantifying the spatial distribution of the seismic hazard.

Wesseloo (2018) takes this one step further and defines a hazard rating based on the same definition for the characteristic volume. The hazard rating is defined as the magnitude with a $15 \%$ probability of exceedance within the equivalent representative volume. This hazard rating definition was chosen to produces similar rating values to the hazard scale originally proposed by Hudyma and Potvin (2004), with which many mines in Australia and Canada are familiar. For the scenario in Figure 16b, this leads to the spatial hazard rating shown in Figure 17b. This approach provides a method for representing the spatial distribution of hazard which is independent of the size of the sub-volume.

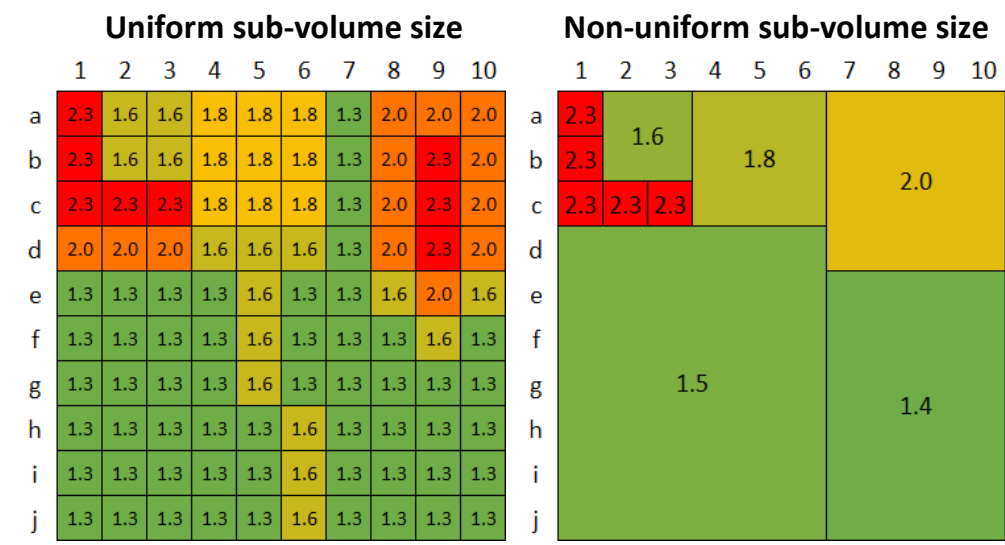

a) a/b normalised to an equivalent representative volume Uniform sub-volume size
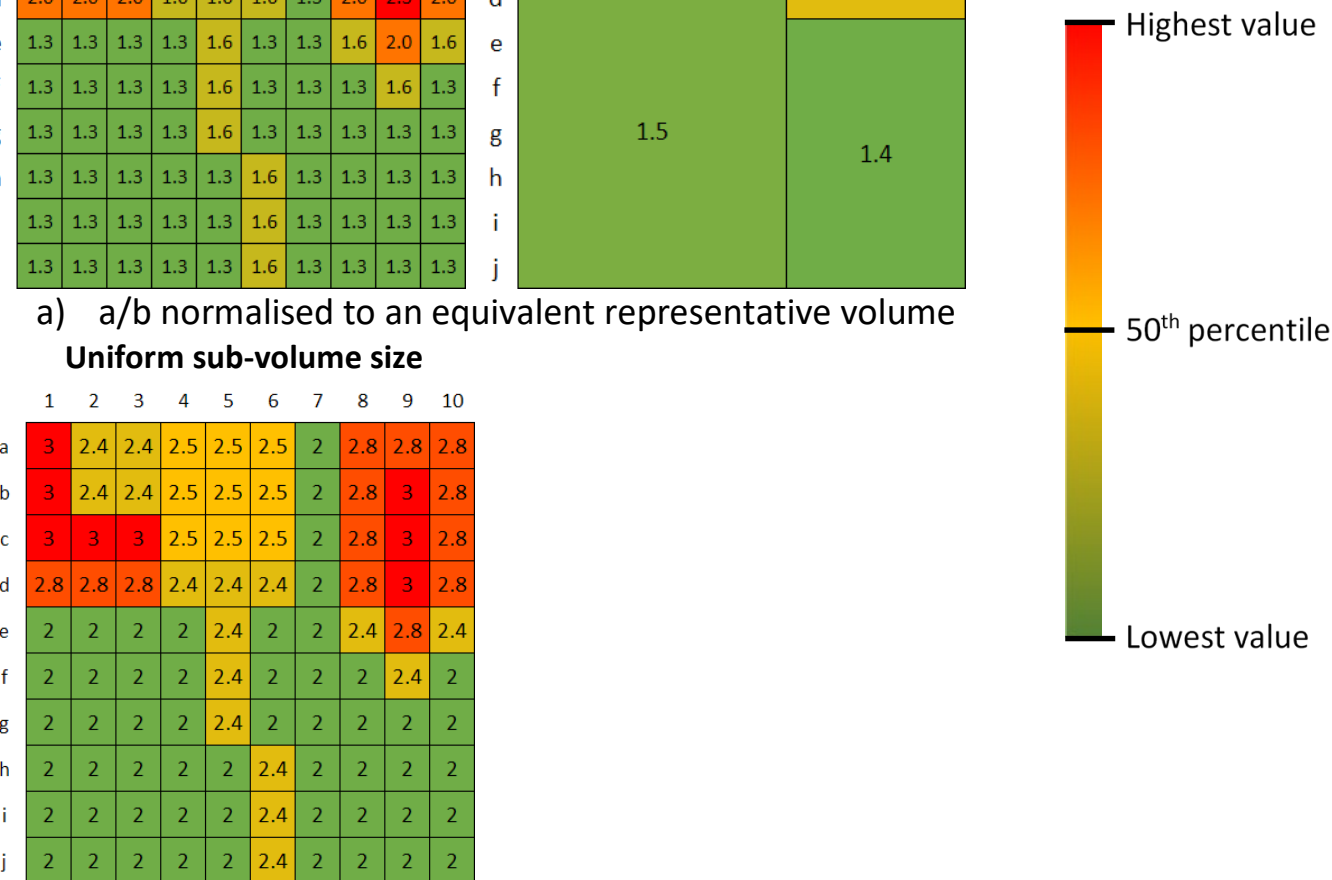

b) hazard rating proposed by Wesseloo (2018)

Figure 17. Hazard rating based equivalent representative volume, independent of the sub-square size 


\section{Time normalisation}

Normalisation of hazard is as important in the time domain as it is in space. To enable direct comparison between hazards of different durations, it is necessary to normalise the calculated probability to the same equivalent timescale. This normalisation can be performed as follows (Wesseloo, 2018):

$$
P_{T n}=1-\left(1-P_{T e}\right)^{\frac{T n}{T e}}
$$

where

$\mathrm{Tn}, \mathrm{Te}=\quad$ the normalised timeframe and the original timeframe, respectively

$\mathrm{PTn}, \mathrm{PTe}=\quad$ the equivalent probabilities expressed for timeframes Tn and Te, respectively

A hazard with a weekly probability of $1 \%$ can be expressed with equivalent annualised values as $1-(1-0.01)^{52}=40 \%$, whilst a hazard with a biennial probability of $50 \%$ can be expressed in equivalent annualised values as $1-(1-0.5)^{0.5}=30 \%$.

Normalisation can be performed to any timescale but short time periods should be avoided however, as it leads to small numbers which, in my experience, are often misinterpreted as small hazards. The use of one year (annualisation) seems a reasonable approach and corresponds with the practice in other branches of engineering, financial risk management and corporate governance (Jonkman et al., 2003, Terbrugge et al., 2006, Stacey et al., 2007, Wesseloo and Read, 2009). Annualisation also allows one to calculate the associated risk and evaluate it against corporate accepted annualised risk levels.

Annualisation of hazard values leads to an 'annual probability', but it is important to note that this should not be interpreted as the probability for a physical year (future or historical). It is the probability value appropriate to the timescale for which the mean seismic rate is applicable, expressed in equivalent annualised terms.

The example calculations following Equation 17 assume that both hazards are present over a long time period. If, for example, a hazard with a weekly probability of $1 \%$ is only present for one week in the year, the yearly hazard would also be $1 \%$. This sometimes leads to misunderstanding in the application of hazard assessments in industry where a short-lived but repeating hazard is sometimes evaluated in isolation.

Seismic hazard in a mine is transient in space and time and, although the seismic hazard at a specific location might be short lived, the hazard is of a repeating nature. For example, the hazard associated with the mining of a single stope might only be present during the time it takes to mine that stope, but, a similar hazard might occur due to the mining of the next stope. Both long-term and repeating hazards can be normalised temporally using Equation 17.

In a previous paper (Wesseloo, 2018), I illustrated this concept with the following fictitious mining scenario that consists of 12 stopes (Figure 18). Each of the stopes is mined for a month during which a seismic response is induced in the indicated annulus around it. The seismicity in this annulus ceases when mining in this stope is complete. During the following month, the next stope is mined with the associated induced seismicity limited to its surrounding annulus. The argument can be further simplified by assuming a constant $b=1$ over the whole volume and the whole year, and by assuming that the total number of events occurring in the annulus of each of the stopes is the same at $\mathrm{N} @-2=1000(a=1)$. 


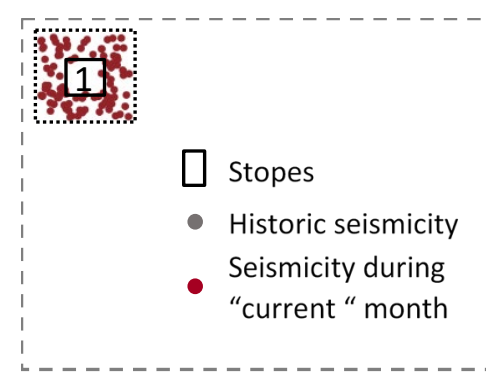

a) Month 1

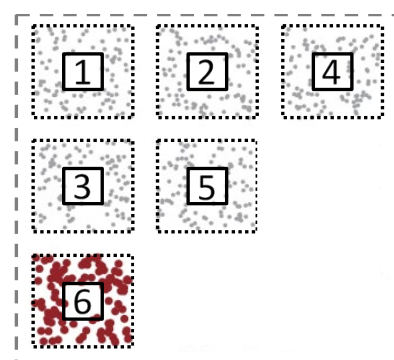

b) Month 6

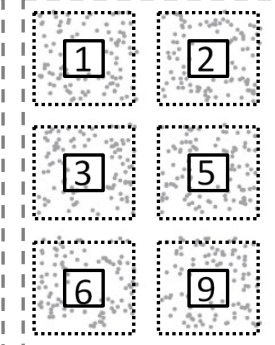

c) Month 12
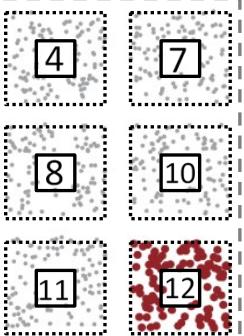

कำ.

Figure 18. Conceptual mine layout for illustrative example, consisting of 12 stopes with an annulus of seismicity induced by the stoping activity

For each stope, the probability of exceeding M2 is $3.92 \%$, and if evaluated in isolation, may be regarded as acceptable. Cumulating the number of events for all 12 stopes, however, results in the total probability of exceeding M2 of $38 \%$. In the year, the company is exposed to the total hazard of $\mathrm{P}\left[\mathrm{M}_{\mathrm{L}}>2\right]=38 \%$, even though each stope only has an individual monthly hazard of $3.9 \%$. If exposing the company to the yearly hazard of $38 \%$ is not acceptable, by implication, it is not acceptable to expose the company to the hazard associated with every one of those stopes individually.

\section{CONCLUDING REMARKS}

The topic of seismic hazard assessment is subject to several misconceptions in the mining industry. This paper addressed some of these misconceptions. The term ' $M_{\max }$ ' is used for several different concepts which appear to add to these misconceptions. To avoid confusion, I suggest that the term ' $M_{\max }$ ' should be reserved for the concept of the largest magnitude physical conditions will allow. Other concepts referred to as ' $M_{\max }$ ' should be referred to by unique names. I propose the use of ' $X_{\max }$ ' for the largest recorded event in a dataset, ' $a / b$ ' for the value of the fitted GR relationship at $N=1$, and, ' $M_{U L}$ ' for the upper asymptote of the FM distribution.

The value of the fitted GR relationship at $\mathrm{N}=1(a / b)$ is sometimes interpreted as the expected largest event. This value is, however, the mode of the distribution of the largest expected magnitude with a $63 \%$ change of being exceeded for the open-ended GR relationship. The probability of exceedance is smaller for truncated GR relationship and depends on the upper limit of the distribution.

The accuracy of methods for estimating $M_{\max }$ is low when $a / b \ll M_{\max }$ and tends to underestimate $M_{\max }$. Reliable results can only be obtained when $M_{\max }$ is based on large datasets, (e.g. N@0>1000 for $M_{\max }=2.5 \mathrm{~N} @ \mathrm{M}>10000$ for $\left.M_{\max }=4.5\right)$.

When assessing the probability of exceeding a specified magnitude $P\left[M>M_{L}\right]$, underestimation of $M_{u L}$ $\left(M_{U L}=M_{\max }-\delta\right)$ leads to larger errors than overestimation of $M_{U L}$ by the same amount $\left(M_{U L}=M_{\max }+\delta\right)$. Underestimating $M_{u L}$, is always optimistic, whilst overestimation is always conservative. For the purpose of hazard assessment, it is therefore prudent to use values for $M_{U L}$ that are deliberately conservative.

Artificial Black Swan Events can be created when $M_{u L}$ underestimates $M_{\max }$. This can occur simply due to the effect of uncertainty. The under-recording of low frequencies due to sensor limitations leads to underestimation of $M_{\max }$ and also leads to artificial Black Swan Events. To combat this problem, sensors which are able to adequately record the lower frequency content of the event sizes expected at the mine, need to be included in the system. In lieu of this, corrections may be applied to the recorded values to compensate for the effect of the sensors under-recording. 
Assessment of the seismic hazard needs to be performed on sub-volumes for which the $b$-value and the event rate can be assumed to be constant.

For comparative hazard evaluation in space and time, both spatial and temporal normalisation are necessary. Normalisation to a characteristic volume equal to a sphere with a $50 \mathrm{~m}$ radius is suggested. Annualisation of hazards are proposed for temporal normalisation.

\section{ACKNOWLEDGEMENT}

I thank William Joughin and Lindsay Linzer for their valuable comments on the manuscript. I also thank all my colleagues at the ACG for their support and, in particular, Yves Potvin, Gerhard Morkel, Kyle Woodward, Dan Cumming-Potvin and Stuart Tierney for fruitful technical discussions. I also would like to thank Paul Harris and Matt Heinsen Egan for always being available to help me with any scripting troubleshooting and app building in mXrap. Thanks also to Josephine Ruddle and Christine Neskudla for proofing my language. The content of this paper has flowed from work done over the span of several years with the support of the mXrap Consortium.

\section{APPENDIX A}

\section{The probability of exceeding an event size of $a / b$ within $n$ events}

For the TGR relationship, the CDF of the event distribution from the magnitude of completeness $m_{\min }$ is then given by

$$
F(M)=1-\frac{1-10^{-b \cdot\left(M-m_{\min }\right)}}{1-10^{-b \cdot\left(M_{U L}-m_{\min }\right)}}
$$

The probability of exceeding an event size of $M_{L}$ within $n$ events is given by (Gibowicz and Kijko, 1994):

$$
F_{\text {max }}(M)=(F(M))^{n}
$$

where:

$F_{\max }(M) \quad-\quad$ cumulative distribution function of the magnitude of the largest event

$F(M)=$ the cumulative probability density function describing the magnitude distribution of event size

The probability of exceeding the value of $a / b$ within $n$ events of magnitude $\geq m_{\min }$ is given by

$$
P\left[X \max \geq \frac{a}{b}\right]=1-F_{\max }\left(\frac{a}{b}\right)=1-F\left(\frac{a}{b}\right)^{n}
$$

And defining $M_{U L}=f \cdot \frac{a}{b}$ leads to

$$
=1-\left(\frac{1-10^{-b \cdot\left(\frac{a}{b}-m_{\min }\right)}}{1-10^{-b \cdot\left(f \cdot \frac{a}{b}-m_{\min }\right)}}\right)^{n}=1-\left(1-\frac{1}{n}\right)^{\mathrm{n}}
$$

This relationship is independent of $m_{\min }$ and can be written in terms of the $a$ value as

$$
P\left[X \max \geq \frac{a}{b}\right]=1-\left(\frac{1-10^{-a}}{1-10^{a \cdot f}}\right)^{10^{a}}
$$

Which, for the open-ended GR relationship reduces to 


$$
P\left[X \max \geq \frac{a}{b}\right]=1-\left(1-10^{-a}\right)^{10^{a}}
$$

\section{The mode of the distribution of $F_{\max }$}

The mode of $f_{\max }(x)$ can be obtained as follows:

$$
\frac{d^{2}}{d x^{2}} F_{\max }(x)=\frac{b^{2} \cdot n \cdot \ln (10)^{2} \cdot\left(A^{n-2}-n \cdot A^{n-2}+10^{b \cdot x} \cdot A^{n-1}\right)}{10^{2 b x}}=0 ; A=\left(1-10^{-b \cdot x}\right)
$$

which reduces to $x=a / b$ for both the open-ended and TGR relationship.

\section{REFERENCES}

Boore, D. (1986). The Effect of Finite Bandwidth on Seismic Scaling Relationships. In Earthquake Source Mechanics. Das, S., Boatwright, J. and Scholz, C.H. (eds.), American Geophysical Union, Washington.

Di Bona, M. and Rovelli, A. (1988). Effects of the Bandwidth Limitation of Stress Drops Estimated from Integrals of the Ground Motion. Bulletin of the Seismological Society of America, 78 (5), 1818-1825.

Financial Times. Financial Times Lexicon [Online]. Financial Times. Available: http://lexicon.ft.com/ [Accessed 22 March 2019 2019].

Gibowicz, S.J. and Kijko, A. (1994). An Introduction to Mining Seismology, San Diego, Academic Press.

Gutenberg, B. and Richter, C.F. (1944). Frequency of Earthquakes in California. Bulletin of the Seismological Society of America, 34 (4), 185-188.

Hudyma, M. and Potvin, Y. (2004). Seismic Hazard in Western Australian Mines. The Journal of The South African Institute of Mining and Metallurgy, 104 (5), 265-275.

Hudyma, M.R. (2008). Analysis and Interpretation of Clusters of Seismic Events in Mines. PhD Thesis, University of Western Australia.

Jonkman, S.N., van Gelder, P.H.A.J.M. and Vrijling, J.K. (2003). An Overview of Quantitative Risk Measures for Loss of Life and Economic Damage. Journal of Hazardous Materials, 99 (1), 1-30.

Kijko, A. (2004). Estimation of the Maximum Earthquake Magnitude, $\mathrm{M}_{\max }$. Pure and Applied Geophysics, $161(8), 1655-1681$.

Kijko, A. (2012). On Bayesian Procedure for Maximum Earthquake Magnitude Estimation. Research in Geophysics, 2 (1).

Kijko, A. and Funk, C. (1994). The Assessment of Seismic Hazards in Mines. The Journal of the South African Institute of Mining and Metallurgy, 94 (4), 179-185.

Kijko, A. and Singh, M. (2011). Statistical Tools for Maximum Possible Earthquake Magnitude Estimation. Acta Geophysica, 59 (4), 674-700.

Lasocki, S. and Urban, P. (2011). Bias, Variance and Computational Properties of Kijko's Estimators of the Upper Limit of Magnitude Distribution, M-Max. Acta Geophysica, 59 (4), 659-673.

Mendecki, A. (2012). Keynote Lecture: Size Distribution of Seismic Events in Mines. Proceedings of Australian Earthquake Engineering Society Conference. Australian Earthquake Engineering Society, pp. 20.

Mendecki, A.J. (2013). Frequency Range, $\log E, \log P$ and Magnitude. Proceedings of Eighth International 
Symposium on Rockbursts and Seismicity in Mines - RaSiM8. Malovichko, A. and Malovichko, D., (eds.), Russia, Geophysical Survey of Russian Academy of Sciences, Mining Institute of Ural Branch of Russian Academy of Sciences, pp. 167-180.

Mining Research Directorate (1996). Canadian Rockburst Research Program 1990-1995, a Comprehensive Summary of Five Years of Collaborative Research on Rockbursting in Hardrock Mines, Canada, CAMIRO Mining Division.

Morkel, I.G. and Wesseloo, J. (2017). The Effect of Sensor Bandwidth Limitations on the Calculation of Seismic Hazard for Mines. Proceedings of Ninth International Symposium on Rocbursts and Seismicity in Mines. Vallejos, J.A., (ed.), Ediarte S.A., pp. 42-49.

Page, R. (1968). Aftershocks and Microaftershocks of the Great Alaska Earthquake of 1964. Bulletin of the Seismological Society of America, 58 (3), 1131-1168.

Potvin, Y., Wesseloo, J., Morkel, I.G., Tierney, S.R., Woodward, K. and Cuello, D. (2019). Management of Seismic Risks in Metalliferous Mines. Proceedings of The Ninth International Conference on Deep and High Stress Mining - Deep Mining 2019. The Southern African Institute of Mining and Metallurgy, pp. in print.

Stacey, T.R., Terbrugge, P.J. and Wesseloo, J. (2007). Risk as a Rock Engineering Design Criterion. Challenges in Deep and High Stress Mining. Potvin, Y., Stacey, T.R. and Hadjigeorgiou, J. (eds.), Australia, Australian Centre for Geomechanics.

Terbrugge, P.J., Wesseloo, J., Venter, J. and Steffen, O.K.H. (2006). A Risk Consequence Approach to Open Pit Slope Design. Journal of the South African Institute of Mining and Metallurgy, 106 (7), 503511.

Utsu, T. (1999). Representation and Analysis of the Earthquake Size Distribution: A Historical Review and Some New Approaches. Pure and Applied Geophysics, 155 (2-4), 509-535.

Wesseloo, J. (2018). The Spatial Assessment of the Current Seismic Hazard State for Hard Rock Underground Mines. Rock Mechanics and Rock Engineering, 51 (6), 1839-1862.

Wesseloo, J. and Read, J. (2009). Acceptance Criteria. Guidelines for Open Pit Slope Design. Stacey, P. and Read, J. (eds.), Australia, CSIRO Publishing.

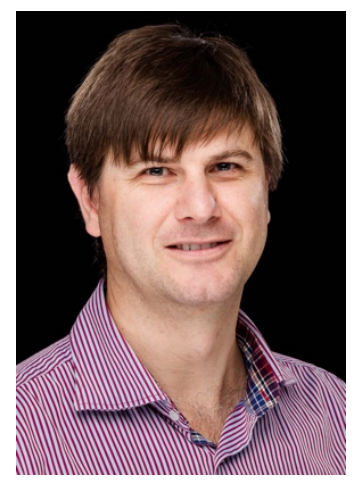

\section{Johan Wesseloo}

Associate Professor

The University of Western Australia

Since 1998, Johan has been involved in geotechnical engineering in the mining industry. He started his career at SRK Consulting, South Africa and joined the Australian Centre for Geomechanics in 2007. His project experience in includes both underground and open pit environments in many countries. Since 2009, much of his work has centred around the different aspects of mining-induced seismicity and rockburst risk management and, with his colleagues, Dr Wesseloo has developed several procedures and methods for seismic analysis for rock engineering purposes and mining seismic hazard assessment 
methods; most featured in the ACG software, mXrap. Johan obtained a BEng (Civil), MEng (Geotechnical) and $\mathrm{PhD}$ (Geotechnical) from The University of Pretoria, South Africa, and is a Fellow of both the Southern African and the Australian Institutes of Mining and Metallurgy. 
